\title{
Arbitrary synthetic dimensions via multiboson dynamics on a one-dimensional lattice
}

\author{
Dali Cheng, ${ }^{1}$ Bo Peng, ${ }^{1}$ Da-Wei Wang, ${ }^{2}$ Xianfeng Chen,,${ }^{1,3}$ Luqi Yuan $\odot,{ }^{1, *}$ and Shanhui Fan ${ }^{4}$ \\ ${ }^{1}$ State Key Laboratory of Advanced Optical Communication Systems and Networks, School of Physics and Astronomy, \\ Shanghai Jiao Tong University, Shanghai 200240, China \\ ${ }^{2}$ Interdisciplinary Center for Quantum Information, State Key Laboratory of Modern Optical Instrumentation, and Zhejiang Province Key \\ Laboratory of Quantum Technology and Device, Department of Physics, Zhejiang University, Hangzhou 310027, Zhejiang Province, China \\ ${ }^{3}$ Shanghai Research Center for Quantum Sciences, Shanghai 201315, China; \\ Jinan Institute of Quantum Technology, Jinan 250101, China; \\ and Collaborative Innovation Center of Light Manipulation and Applications, Shandong Normal University, Jinan 250358, China \\ ${ }^{4}$ Ginzton Laboratory and Department of Electrical Engineering, Stanford University, Stanford, California 94305, USA
}

(Received 22 March 2021; accepted 23 June 2021; published 19 July 2021)

\begin{abstract}
The synthetic dimension, a research topic of both fundamental significance and practical applications, has been attracting increasing attention in recent years. In this paper, we propose a theoretical framework to construct arbitrary synthetic dimensions, or $N$-boson synthetic lattices, using multiple bosons on one-dimensional lattices. We show that a one-dimensional lattice hosting $N$ indistinguishable bosons can be mapped to a single boson on an $N$-dimensional lattice with high symmetry. Band structure analyses on this $N$-dimensional lattice can then be mathematically performed to predict the existence of exotic eigenstates and the motion of $N$-boson wave packets. As illustrative examples, we demonstrate the edge states in two-boson Su-Schrieffer-Heeger synthetic lattices without interactions, interface states in two-boson Su-Schrieffer-Heeger synthetic lattices with interactions, and weakly bound triplon states in three-boson tight-binding synthetic lattices with interactions. The interface states and weakly bound triplon states have not been thoroughly understood in previous research. Our proposed theoretical framework hence provides an interesting perspective to explore the multiboson dynamics on lattices with boson-boson interactions, and opens up a future avenue in the field of multiboson manipulation in quantum engineering.
\end{abstract}

DOI: 10.1103/PhysRevResearch.3.033069

\section{INTRODUCTION}

Dimensionality is one of the most important concepts in modern physics. In condensed-matter physics, systems with different dimensionalities exhibit vastly different behaviors. Notable examples include topological insulators with protected surface states [1-6] and superconducting electronic gases confined in quantum structures [7-11], which all exhibit significant dependence on system dimensionality. To explore phenomena unique to high-dimensional physics, the artificial synthesis of extra dimensions in low-dimensional platforms has attracted great interest in recent years [12-16], partly because experimental platforms, including dielectrics [17], plasmons [18,19], atoms [13,20,21], and magnetons [22], feature relatively convenient fabrication and manipulation in low dimensions. By connecting internal degrees of freedom of particles to form apparent artificial lattices [15], one can successfully construct synthetic dimensions which, together

\footnotetext{
*yuanluqi@sjtu.edu.cn

Published by the American Physical Society under the terms of the Creative Commons Attribution 4.0 International license. Further distribution of this work must maintain attribution to the author(s) and the published article's title, journal citation, and DOI.
}

with spatial dimensions, make the dimensionality of a physical system beyond that of real space. This focus on synthetic dimensions is of fundamental significance in physics, and also holds promising applications in the field of optical communication and quantum information processing [23-27].

Quantum many-body physics, as an important research subject in quantum physics, receives intensive study, with its counterparts also greatly explored by quantum simulations in optical platforms [28-30]. As an outstanding example, repulsively bound boson pairs, also called doublons, have been unveiled as a result of the Bose-Hubbard Hamiltonian [31]. Following this important discovery, quantum problems of two-particle states with one-dimensional (1D) interactive Hamiltonians, such as the tight-binding Bose-Hubbard model [32]; the tight-binding Bose-Hubbard model with a parabolic potential [33], with a small impurity potential [34], and with nonlocal interactions [35-37]; and the SuSchrieffer-Heeger (SSH) Bose-Hubbard model with nonlocal interactions [38-42]; as well as two-particle problems with Bloch oscillation in an external electrical field [43-45], have been investigated with very broad interest in both condensedmatter and optical societies. In particular, when two correlated indistinguishable boson dynamics is under exploration, the $1 D-2 D$ mapping approach $[32,34,35,37,41,42,45]$ has been used to convert the two-boson dynamics on 1D lattices into the single-particle dynamics on two-dimensional (2D) lattices. 
This mapping method significantly facilitates the theoretical analysis and deepens our understanding of two-boson interacting dynamics in the quantum regime.

Inspired by the works mentioned above, in this paper, we propose to construct arbitrary synthetic dimensions with multiple bosons in a 1D array, i.e., an $N$-boson synthetic lattice, by generalizing the $1 \mathrm{D}-2 \mathrm{D}$ mapping method to a $1 \mathrm{D}-\mathrm{N}$-dimensional counterpart. After properly choosing the symmetry restriction of the wave function, this $N$-dimensional synthetic space with $N$ indistinguishable bosons features periodicity along each of its synthetic dimensions, making it possible to predict the $\mathrm{N}$-boson dynamics by utilizing the well-established band structure approach. We also note some related research [46-49] providing another method of constructing synthetic dimensions with multiple bosons. In these previous works, the extra degrees of freedom originate in the boson numbers in the system. In our theoretical framework, however, they come from the lattice site indices of the bosons in the 1D array. As for demonstrations, we provide examples exploring multiparticle physics with extended nonlinearity including the two-boson case supporting the topological interface states, as well as the three-boson case predicting the boson blockade effects in three dimensions. Our work hence points out a fundamentally different perspective for studying many-body dynamics with nonlinearity from a physical picture with synthetic dimensions, which also shows potential applications in quantum simulations and quantum information processing with synthetic dimensions in optical systems.

The remaining parts of this paper are organized as follows. Section II describes the fundamental theoretical framework of the $N$-boson synthetic lattice that will be adopted throughout this paper. Sections III-V give examples addressing the richness of various systems where our theoretical framework is applicable. Section VI discusses some possible experimental implementations followed by a brief conclusion.

\section{THEORETICAL FRAMEWORK}

We start to establish our theoretical framework by examining two indistinguishable bosons on an infinite 1D tightbinding lattice, and then move on to the $N$-boson case. The two-boson case has been addressed in several previous studies as the 1D-2D mapping approach [32,34,35,37,41,42,45]. Here we first reiterate this approach in a more explicit and systematic way. The Hamiltonian of the system is

$$
H=g \sum_{k=-\infty}^{+\infty}\left(b_{k}^{\dagger} b_{k+1}+b_{k+1}^{\dagger} b_{k}\right),
$$

where $g$, a real number, is the coupling strength between neighboring sites, and $b_{k}\left(b_{k}^{\dagger}\right)$ is the annihilation (creation) operator of bosons on the $k$ th lattice site as shown in Fig. 1(a). We take $\hbar=1$ throughout the paper for simplicity. In Schrödinger's picture, the two-boson state is

$$
|\phi(t)\rangle=\frac{1}{\sqrt{2}} \sum_{m=-\infty}^{+\infty} \sum_{n=-\infty}^{+\infty} v_{m n}(t) b_{m}^{\dagger} b_{n}^{\dagger}|0\rangle,
$$

where $|0\rangle$ is the vacuum state. By substituting Eqs. (1) and (2) into the Schrödinger equation $i|\dot{\phi}\rangle=H|\phi\rangle$, the differential

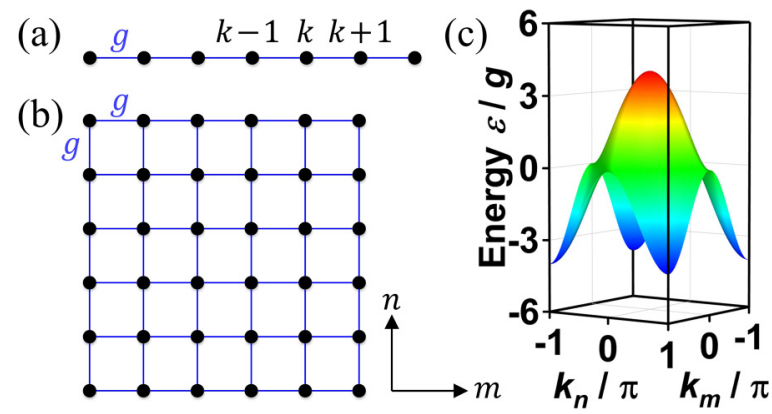

FIG. 1. The two-boson tight-binding synthetic lattice without interactions. (a) A 1D tight-binding lattice with coupling strength $g$. (b) The two-boson synthetic lattice with exchange symmetry restriction on the wave function $v_{m n}(t)$. (c) 2D band structure of the lattice in (b) in the first Brillouin zone.

equation satisfied by $v_{m n}$ is derived as

$$
\begin{aligned}
i\left(\dot{v}_{m n}+\dot{v}_{n m}\right)= & g\left(v_{m-1, n}+v_{m+1, n}+v_{m, n-1}+v_{m, n+1}\right. \\
& \left.+v_{n-1, m}+v_{n+1, m}+v_{n, m-1}+v_{n, m+1}\right) .
\end{aligned}
$$

Here, the conventionally standard restriction on $v_{m n}$ is to follow the exchange symmetry; i.e., we let $v_{m n}=v_{n m}$. With this restriction,

$$
\begin{aligned}
\left\langle 0\left|b_{m} b_{n}\right| \phi\right\rangle & =\frac{1}{\sqrt{2}} \sum_{m^{\prime}=-\infty}^{+\infty} \sum_{n^{\prime}=-\infty}^{+\infty} v_{m^{\prime} n^{\prime}}\left\langle 0\left|b_{m} b_{n} b_{m^{\prime}}^{\dagger} b_{n^{\prime}}^{\dagger}\right| 0\right\rangle \\
& =\frac{v_{m n}+v_{n m}}{\sqrt{2}}=\sqrt{2} v_{m n},
\end{aligned}
$$

and the square of the modulus of $v_{m n}$ can then be identified as the second-order correlation function of the two-boson state:

$$
\left|v_{m n}\right|^{2}=\frac{1}{2}\left\langle\phi\left|b_{n}^{\dagger} b_{m}^{\dagger}\right| 0\right\rangle\left\langle 0\left|b_{m} b_{n}\right| \phi\right\rangle=\frac{1}{2}\left\langle\phi\left|b_{n}^{\dagger} b_{m}^{\dagger} b_{m} b_{n}\right| \phi\right\rangle .
$$

Also, the probability amplitude that one boson is observed on the $m$ th lattice site and the other on the $n$th site $(m \leqslant n)$ is

$$
u_{m n}=\left\{\begin{array}{ll}
\left\langle 1_{m} 1_{n} \mid \phi\right\rangle=\sqrt{2} v_{m n}=\sqrt{2} v_{n m} & \text { if } m<n \\
\left\langle 2_{m} \mid \phi\right\rangle=v_{m m} & \text { if } m=n
\end{array} .\right.
$$

Since we have required that $v_{m n}=v_{n m}$, Eq. (3) simplifies to

$$
i \dot{v}_{m n}=g\left(v_{m-1, n}+v_{m+1, n}+v_{m, n-1}+v_{m, n+1}\right),
$$

which shows that the dynamics of $v_{m n}$ is mathematically equivalent to the single-particle dynamics on a $2 \mathrm{D}$ tightbinding lattice. This lattice, referred to as a two-boson synthetic lattice, is illustrated in Fig. 1(b), and the process of constructing such two-boson synthetic lattice can be denoted as 1D-2D mapping.

The procedure for the construction of such synthetic lattice can be generalized for $N$-boson states. We consider the tightbinding Hamiltonian of Eq. (1) operating on an $N$-boson state:

$$
|\phi(t)\rangle=\frac{1}{\sqrt{N !}} \sum_{\lambda_{1}=-\infty}^{+\infty} \cdots \sum_{\lambda_{N}=-\infty}^{+\infty}\left[v_{\lambda_{1}, \ldots, \lambda_{N}}(t) b_{\lambda_{1}}^{\dagger} \cdots b_{\lambda_{N}}^{\dagger}\right]|0\rangle .
$$


From the Schrödinger equation, we can calculate that

$$
\begin{aligned}
i \sum_{\text {permu }} \dot{v}_{\lambda_{1}, \ldots, \lambda_{N}}= & g \sum_{\text {permu }} \sum_{l=1}^{N}\left(v_{\lambda_{1}, \ldots, \lambda_{l-1}, \lambda_{l}+1, \lambda_{l+1}, \ldots, \lambda_{N}}\right. \\
& \left.+v_{\lambda_{1}, \ldots, \lambda_{l-1}, \lambda_{l}-1, \lambda_{l+1}, \ldots, \lambda_{N}}\right),
\end{aligned}
$$

where $\sum_{\text {permu }}(\cdots)$ denotes the permutation sum by rerranging the sequence $\lambda_{1}, \ldots, \lambda_{N}$. Similarly here, the wave function is additionally restricted by the exchange symmetry that $v_{\lambda_{1}, \ldots, \lambda_{N}}$ remains constant after exchanging any two subscripts $\lambda_{i}$ and $\lambda_{j}$. In this case,

$$
\left\langle 0\left|b_{\lambda_{1}} \ldots b_{\lambda_{N}}\right| \phi\right\rangle=\frac{\sum_{\text {permu }} v_{\lambda_{1}, \ldots, \lambda_{N}}}{\sqrt{N !}}=\sqrt{N !} v_{\lambda_{1}, \ldots, \lambda_{N}},
$$

and the square of the modulus of $v_{\lambda_{1}, \ldots, \lambda_{N}}$ is the $N$ th-order correlation function:

$$
\left|v_{\lambda_{1}, \ldots, \lambda_{N}}\right|^{2}=\frac{1}{N !}\left\langle\phi\left|b_{\lambda_{N}}^{\dagger} \cdots b_{\lambda_{1}}^{\dagger} b_{\lambda_{1}} \cdots b_{\lambda_{N}}\right| \phi\right\rangle
$$

Using $v_{\lambda_{1}, \ldots, \lambda_{N}}$, the probability amplitude that $\xi_{i}$ bosons are observed on the $\mu_{i}$-th lattice site $\left(\xi_{i}>0, \sum_{i} \xi_{i}=N\right.$, and $\mu_{i}<$ $\mu_{j}$ if $i<j$ ) can be expressed as

$$
\begin{aligned}
& \underbrace{u_{\xi_{1}}^{\mu_{1}, \mu_{1}, \ldots, \mu_{1}}, \ldots, \underbrace{\mu_{i}, \mu_{i}, \ldots, \mu_{i}}_{\xi_{i}}, \cdots}_{N} \\
& =\left\langle\xi_{1 \mu_{1}} \cdots \xi_{i \mu_{i}} \cdots \mid \phi\right\rangle \\
& =\sqrt{\frac{N !}{\prod_{i} \xi_{i} !}} \underbrace{v_{N}^{\mu_{1}, \mu_{1}, \ldots, \mu_{1}}}_{\xi_{1}}, \ldots, \underbrace{\mu_{i}, \mu_{i}, \ldots, \mu_{i}}_{\xi_{i}}, \cdots
\end{aligned}
$$

Because of the exchange symmetry, Eq. (9) can finally be simplified to

$$
\begin{aligned}
& i \dot{v}_{\lambda_{1}, \lambda_{2}, \ldots, \lambda_{N}} \\
& =g\left[\left(v_{\lambda_{1}+1, \lambda_{2}, \ldots, \lambda_{N}}+v_{\lambda_{1}, \lambda_{2}+1, \ldots, \lambda_{N}}+\cdots+v_{\lambda_{1}, \lambda_{2}, \ldots, \lambda_{N}+1}\right)\right. \\
& \left.\quad+\left(v_{\lambda_{1}-1, \lambda_{2}, \ldots, \lambda_{N}}+v_{\lambda_{1}, \lambda_{2}-1, \ldots, \lambda_{N}}+\cdots+v_{\lambda_{1}, \lambda_{2}, \ldots, \lambda_{N}-1}\right)\right] .
\end{aligned}
$$

Equation (13) is mathematically identical to the coupledmode equations [50] for the wave evolution in an isotropic lattice defined in $N$ dimensions, thus giving the $N$-boson synthetic lattice with $v_{\lambda_{1}, \ldots, \lambda_{N}}$ being the lattice sites.

In deriving Eq. (13), we assume that the wave function $v_{\lambda_{1}, \ldots, \lambda_{N}}$ satisfies exchange symmetry. For the same quantum state of Eq. (8), other restrictions of the wave function $v_{\lambda_{1}, \ldots, \lambda_{N}}$ are also possible. For example, we may also choose $v_{\lambda_{1}, \ldots, \lambda_{N}}$ to be equal to zero except in the region $\lambda_{1} \leqslant \lambda_{2} \leqslant$ $\cdots \leqslant \lambda_{N}$. With this alternative choice, however, Eq. (13) no longer holds, and $v_{\lambda_{1}, \ldots, \lambda_{N}}$ 's appear to be connected to their neighboring sites with nonuniform coupling strengths (see Appendix A). In fact, the uniformity of the lattice as described by Eq. (13) is closely related to imposing the exchange symmetry on the wave function.

The uniformity of the lattice as described in Eq. (13) allows the definition of a band structure in the synthetic space. The steady state solution of Eq. (13) corresponds to the following eigenvalue problem:

$$
\begin{aligned}
& \varepsilon_{\text {tight-binding }}^{N \text {-dimension }} v_{\lambda_{1}, \lambda_{2}, \ldots, \lambda_{N}} \\
& =g\left[\left(v_{\lambda_{1}+1, \lambda_{2}, \ldots, \lambda_{N}}+v_{\lambda_{1}, \lambda_{2}+1, \ldots, \lambda_{N}}+\cdots+v_{\lambda_{1}, \lambda_{2}, \ldots, \lambda_{N}+1}\right)\right. \\
& \left.\quad+\left(v_{\lambda_{1}-1, \lambda_{2}, \ldots, \lambda_{N}}+v_{\lambda_{1}, \lambda_{2}-1, \ldots, \lambda_{N}}+\cdots+v_{\lambda_{1}, \lambda_{2}, \ldots, \lambda_{N}-1}\right)\right] .
\end{aligned}
$$

Since the coupling strengths are uniform in this synthetic lattice along all of the $N$ dimensions $\left(\lambda_{1}, \ldots, \lambda_{N}\right)$, we can define an $N$-boson band structure analytically:

$$
\varepsilon_{\text {tight-binding }}^{N \text {-dimension }}(\mathbf{k})=2 g \sum_{i=1}^{N} \cos k_{i},
$$

where $\mathbf{k}=\left[k_{1}, \ldots, k_{N}\right]^{\mathrm{T}}$ and $k_{i}$ is the wave vector along the $\lambda_{i}$ direction in Eq. (14). The group velocity of the particle wave packet in the synthetic lattice associated with a specific $N$-boson state can be calculated using the band structure by computing the derivative of the energy spectrum with respect to the wave vectors. One notices that the band structure for a 1D tight-binding lattice is $\varepsilon_{\text {tight-binding }}^{1 \mathrm{D}}(k)=2 g \cos k$ [51] so that $\varepsilon_{\text {tight-binding }}^{N \text {-dimenion }}(\mathbf{k})=\sum_{i=1}^{N} \varepsilon_{\text {tight-binding }}^{1 \mathrm{D}}\left(k_{i}\right)$. This result is consistent with the physical intuition that, without the boson-boson interaction, $N$ bosons evolve independently so the $N$-boson dynamics can be understood from the singleboson dynamics. On the other hand, when the boson-boson interaction is involved, the $N$-boson dynamics can no longer be simply derived from the one-boson dynamics. In later sections of this paper, we shall study examples with nonlinear boson-boson interactions, where the $N$-boson synthetic lattice shows great convenience in predicting some intriguing phenomena.

To confirm the predictions made by the $N$-boson synthetic lattice and the corresponding band structure analysis, we can also perform numerical simulations by solving Eq. (13) in the presence of external excitation. Based on the input-output formalism [52], when $\xi_{i}$ bosons are excited on the $\mu_{i}$-th lattice site $\left(\xi_{i}>0, \sum_{i} \xi_{i}=N\right.$, and $\mu_{i}<\mu_{j}$ if $\left.i<j\right)$, a source term $\eta(t)$ should be added to the right side of Eq. (13) if $\lambda_{1}, \ldots, \lambda_{N}$ is a permutation of $\underbrace{\mu_{1}, \mu_{1}, \ldots, \mu_{1}}_{\xi_{1}}, \ldots, \underbrace{\mu_{i}, \mu_{i}, \ldots, \mu_{i}}_{\xi_{i}}, \cdots$. Here $\eta(t)$, the temporal profile of the boson excitation, takes the form of a modulated Gaussian envelope,

$$
\eta(t)=\eta_{0} \exp \left[-\frac{\left(t-t_{0}\right)^{2}}{\tau^{2}}\right] \exp (-i \Delta \varepsilon t), \quad t \geqslant 0,
$$

where $\eta_{0}$ is the normalization coefficient, $t_{0}$ and $\tau$ are the center and the width of the Gaussian pulse respectively, and $\Delta \varepsilon$ is the excitation energy. Finally, the multiboson excitation probabilities can be calculated according to Eq. (12), and the average boson number on the $k$ th lattice site of the hosting 1D tight-binding chain [Fig. 1(a)] can be determined by

$$
N_{k}(t)=\left\langle\phi(t)\left|b_{k}^{\dagger} b_{k}\right| \phi(t)\right\rangle .
$$

Some typical simulation results of the two-boson dynamics with Hamiltonian equation (1) are displayed in Fig. 2.

Before ending this section, we note that, for illustration purposes, the synthetic lattice described in this section is 
(a)

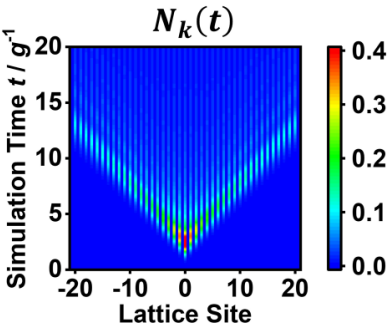

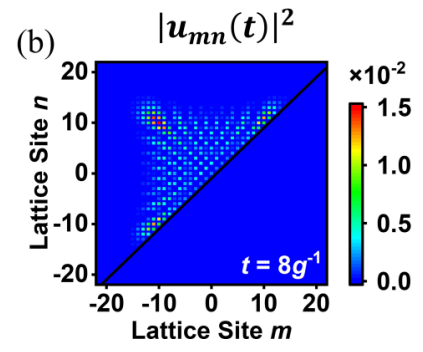

FIG. 2. Simulation results of two-boson dynamics in the twoboson tight-binding synthetic lattice without interactions. (a) Average boson numbers on lattice sites and (b) the distribution of boson excitation probabilities when two bosons are excited on the 0th site simultaneously. $\Delta \varepsilon=0, t_{0}=2 g^{-1}$, and $\tau^{2}=1.5 g^{-2}$ in this figure.

rather simple with only real-valued, nearest-neighbor coupling and no interaction. However, more complex behaviors including nontrivial topological and interacting features can be created in the $N$-boson synthetic lattice based on the same theoretical framework as introduced here. To highlight these more complex features, in the following, we discuss two bosons on an SSH lattice without/with interactions (Secs. III and IV), and two/three bosons on a tight-binding extended Bose-Hubbard lattice (Appendix B and Sec. V).

\section{TWO BOSONS ON AN SSH LATTICE WITHOUT INTERACTIONS}

As the first illustration of interesting topological behaviors in the $\mathrm{N}$-boson synthetic lattice, here we examine the twoboson dynamics on a 1D SSH lattice. The 1D SSH model is perhaps one of the simplest models that exhibit a nontrivial energy band topology [53], and has been widely studied [54-59]. Here we show that dynamics of two noninteracting bosons on a 1D SSH lattice can be mapped to a 2D SSH model. A 1D SSH lattice, as shown in Fig. 3(a), features alternating coupling strengths of $g_{1}$ and $g_{2}$, and consequently
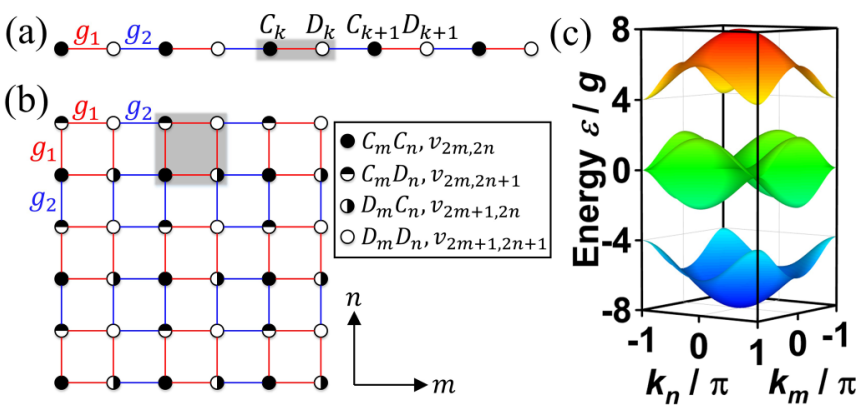

FIG. 3. The two-boson SSH synthetic lattice without interactions. (a) A $1 \mathrm{D} \mathrm{SSH}$ lattice, where black spots are sites $C_{k}$ 's, white spot are sites $D_{k}$ 's, red lines are intracell coupling $g_{1}$, and blue lines are intercell coupling $g_{2}$. One unit cell contains two sites, shaded in gray. (b) The two-boson synthetic lattice with the exchange symmetry restriction on the wave function $v_{2 m, 2 n}(t), v_{2 m, 2 n+1}(t), v_{2 m+1,2 n}(t)$, and $v_{2 m+1,2 n+1}(t)$. One unit cell contains four sites, shaded in gray. (c) 2D band structure of the lattice in (b) in the first Brillouin zone with coupling strengths $g_{1}=3 g, g_{2}=g$.

there are two types of lattice sites on the chain which are denoted as $C_{k}$ and $D_{k}$ in the $k$ th unit cell, respectively. $g_{1}$ is referred to as intracell coupling and $g_{2}$ as intercell coupling. The corresponding Hamiltonian and the two-boson state under consideration are expressed by

$$
\begin{array}{r}
H=g_{1} \sum_{k=-\infty}^{+\infty}\left(c_{k}^{\dagger} d_{k}+d_{k}^{\dagger} c_{k}\right)+g_{2} \sum_{k=-\infty}^{+\infty}\left(c_{k+1}^{\dagger} d_{k}+d_{k}^{\dagger} c_{k+1}\right), \\
|\phi(t)\rangle=\frac{1}{\sqrt{2}} \sum_{m=-\infty}^{+\infty} \sum_{n=-\infty}^{+\infty}\left[v_{2 m, 2 n}(t) c_{m}^{\dagger} c_{n}^{\dagger}+v_{2 m, 2 n+1}(t) c_{m}^{\dagger} d_{n}^{\dagger}\right. \\
\left.\quad+v_{2 m+1,2 n}(t) d_{m}^{\dagger} c_{n}^{\dagger}+v_{2 m+1,2 n+1}(t) d_{m}^{\dagger} d_{n}^{\dagger}\right]|0\rangle
\end{array}
$$

where $c_{k}$ and $d_{k}\left(c_{k}^{\dagger}\right.$ and $\left.d_{k}^{\dagger}\right)$ are annihilation (creation) operators of bosons on the $C_{k}$ and $D_{k}$ sites, respectively. Substituting Eqs. (18) and (19) into the Schrödinger equation $i|\dot{\phi}\rangle=H|\phi\rangle$, we obtain the differential equations:

$$
\begin{aligned}
& i\left(\dot{v}_{2 m, 2 n}+\dot{v}_{2 n, 2 m}\right) \\
&= g_{1}\left(v_{2 m, 2 n+1}+v_{2 n, 2 m+1}+v_{2 m+1,2 n}+v_{2 n+1,2 m}\right) \\
& \quad \quad g_{2}\left(v_{2 m, 2 n-1}+v_{2 n, 2 m-1}+v_{2 m-1,2 n}+v_{2 n-1,2 m}\right), \\
& i\left(\dot{v}_{2 m+1,2 n+1}+\dot{v}_{2 n+1,2 m+1}\right) \\
&= g_{1}\left(v_{2 m, 2 n+1}+v_{2 n, 2 m+1}+v_{2 m+1,2 n}+v_{2 n+1,2 m}\right) \\
& \quad+g_{2}\left(v_{2 m+2,2 n+1}+v_{2 n+2,2 m+1}+v_{2 m+1,2 n+2}\right. \\
& \quad\left.+v_{2 n+1,2 m+2}\right), \\
& i\left(\dot{v}_{2 m, 2 n+1}+\dot{v}_{2 n+1,2 m}\right) \\
&=g_{1}\left(v_{2 m, 2 n}+v_{2 n, 2 m}+v_{2 m+1,2 n+1}+v_{2 n+1,2 m+1}\right) \\
& \quad+g_{2}\left(v_{2 m, 2 n+2}+v_{2 n+2,2 m}+v_{2 m-1,2 n+1}\right. \\
&\left.\quad+v_{2 n+1,2 m-1}\right) .
\end{aligned}
$$

Now we apply the exchange symmetry restriction of the wave function $\left(v_{m n}=v_{n m}\right.$ as discussed in Sec. II), and Eq. (20) can then be transformed into

$$
\begin{aligned}
i \dot{v}_{2 m, 2 n}= & g_{1}\left(v_{2 m, 2 n+1}+v_{2 m+1,2 n}\right) \\
& +g_{2}\left(v_{2 m, 2 n-1}+v_{2 m-1,2 n}\right), \\
i \dot{v}_{2 m, 2 n+1}= & g_{1}\left(v_{2 m, 2 n}+v_{2 m+1,2 n+1}\right) \\
& +g_{2}\left(v_{2 m, 2 n+2}+v_{2 m-1,2 n+1}\right), \\
i \dot{v}_{2 m+1,2 n}= & g_{1}\left(v_{2 m, 2 n}+v_{2 m+1,2 n+1}\right) \\
& +g_{2}\left(v_{2 m+2,2 n}+v_{2 m+1,2 n-1}\right), \\
i \dot{v}_{2 m+1,2 n+1}= & g_{1}\left(v_{2 m, 2 n+1}+v_{2 m+1,2 n}\right) \\
& +g_{2}\left(v_{2 m+2,2 n+1}+v_{2 m+1,2 n+2}\right) .
\end{aligned}
$$

Again, this exchange symmetry is not a physically necessary restriction on the wave function, yet it is mathematically essential in obtaining Eq. (21). Equation (21) gives the twoboson synthetic lattice as illustrated in Fig. 3(b), which corresponds to a standard 2D SSH model [60]. Mathematically, this lattice features translational symmetry along both 

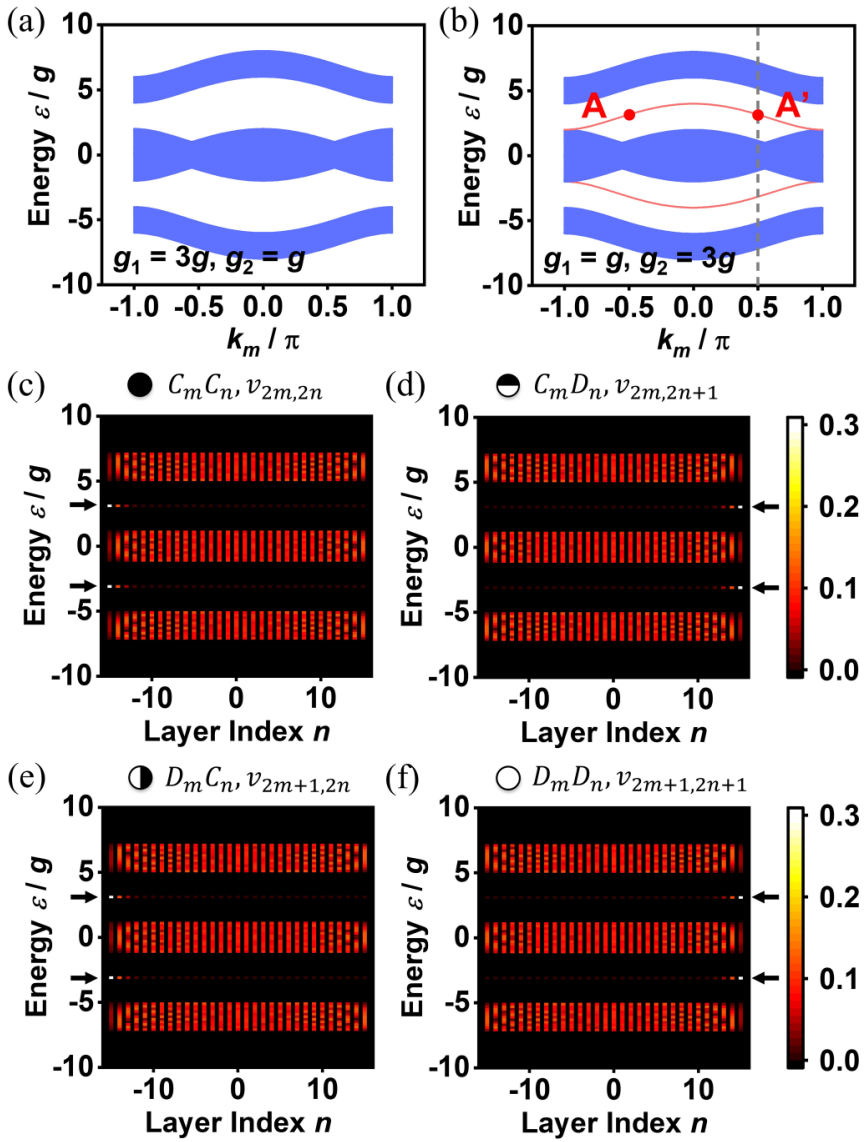

FIG. 4. Projected band structures and eigenstate distributions of an SSH stripe. (a), (b) Projected band structures of an SSH stripe with coupling strengths $\left(g_{1}, g_{2}\right)=(3 g, g)$ or $(g, 3 g)$. Edge states are denoted by red lines in (b). (c)-(f) Eigenstate distributions of (c) $C_{m} C_{n}$-type, (d) $C_{m} D_{n}$-type, (e) $D_{m} C_{n}$-type, and (f) $D_{m} D_{n}$-type lattice sites corresponding to the band structures (b) with $k_{m}=0.5 \pi$ [gray dash in (b)]. Edge modes are indicated by black arrows. $-\infty<m<$ $+\infty$ and $-15 \leqslant n \leqslant 15$ in this figure.

the $m$ and $n$ axes with period 2, and its band structure can be analytically obtained as

$$
\begin{aligned}
\varepsilon_{\mathrm{SSH}}^{2 \mathrm{D}}\left(k_{m}, k_{n}\right)= & \pm \sqrt{g_{1}^{2}+g_{2}^{2}+2 g_{1} g_{2} \cos k_{m}} \\
& \pm \sqrt{g_{1}^{2}+g_{2}^{2}+2 g_{1} g_{2} \cos k_{n}},
\end{aligned}
$$

as plotted in Fig. 3(c) [61]. Four different bands are observed as one unit cell in the two-boson synthetic lattice contains four lattice sites: $v_{2 m, 2 n}, v_{2 m, 2 n+1}, v_{2 m+1,2 n}$, and $v_{2 m+1,2 n+1}$. The band structure of Eq. (22) can also be expressed as

$$
\varepsilon_{\mathrm{SSH}}^{2 \mathrm{D}}\left(k_{m}, k_{n}\right)=\varepsilon_{\mathrm{SSH}}^{1 \mathrm{D}}\left(k_{m}\right)+\varepsilon_{\mathrm{SSH}}^{1 \mathrm{D}}\left(k_{n}\right),
$$

where $\varepsilon_{\mathrm{SSH}}^{1 \mathrm{D}}(k)= \pm \sqrt{g_{1}^{2}+g_{2}^{2}+2 g_{1} g_{2} \cos k}$, because no boson-boson interaction is considered in Hamiltonian equation (18) [62].

A 2D SSH lattice exhibits topological features since edge modes exist in the nontrivial phase $\left(g_{1}<g_{2}\right)$ because of nonzero Berry connection and Zak phase [61]. Here we examine an SSH stripe which is infinite along the $m$ axis but has finite width along the $n$ axis $(-15 \leqslant n \leqslant 15)$. (Strictly
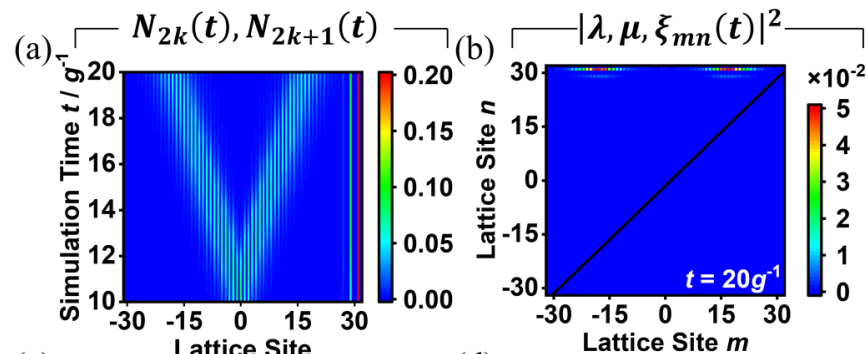

(c)
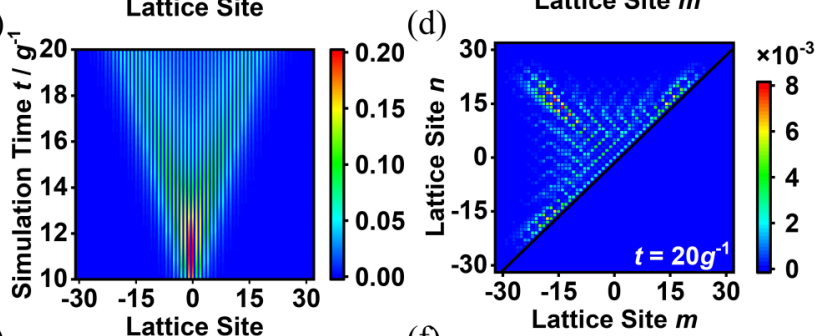

(e)
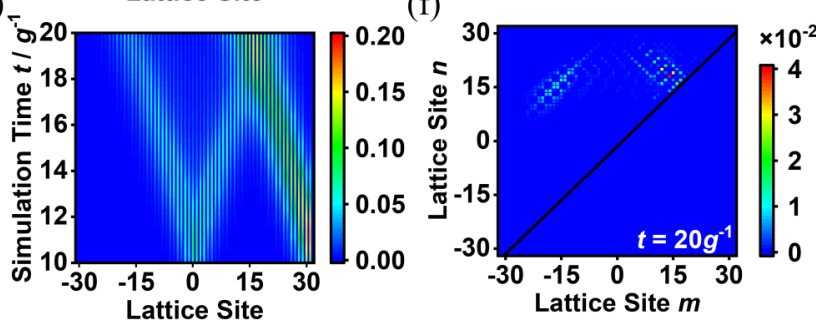

FIG. 5. Simulation results of edge modes in the two-boson SSH synthetic lattice without interactions. (a), (b) The wave packet evolution after exciting two bosons on sites $C_{0}$ and $D_{15}$, respectively, with $g_{1}=g, g_{2}=3 g$, and $\Delta \varepsilon=3.16 g$. (c), (d) The wave packet evolution after exciting two bosons on site $C_{0}$ simultaneously with $g_{1}=g, g_{2}=3 g$, and $\Delta \varepsilon=0$. (e), (f) The wave packet evolution after exciting two bosons on sites $C_{0}$ and $D_{15}$, respectively, with $g_{1}=3 g$, $g_{2}=g$, and $\Delta \varepsilon=0 . t_{0}=10 g^{-1}$ and $\tau^{2}=10 g^{-2}$ in this figure.

speaking, the trivial/nontrivial phase transition point is not exactly at $g_{1}=g_{2}$, depending on the stripe width, but takes $g_{1}=g_{2}$ in the limit of infinite stripe width [61].) One notes that such an SSH stripe is not physically implementable by our platform of two bosons on a 1D lattice, since the wave function exchange symmetry requires that the lattice should have reflection symmetry along the $m=n$ axis, yet this SSH stripe is still a useful mathematical construct for analyzing edge modes. In Figs. 4(a) and 4(b) the projected band structures are plotted in the topologically trivial phase $\left(g_{1}=3 g, g_{2}=g\right)$ and nontrivial phase $\left(g_{1}=g, g_{2}=3 g\right)$, respectively. The bulk modes (drawn in blue) can be viewed as Fig. 3(c) after the band structure is projected onto the $k_{m}$ axis. In the nontrivial phase, edge modes (red lines) are clearly shown in the band gaps. Eigenstate distribution analysis further confirms that these isolated modes are edge modes, since the corresponding eigenstates are localized at lattice sites whose indices $n$ are \pm 15 , i.e., on the edges of the SSH stripe, as indicated by black arrows in Figs. 4(c)-4(f).

Although the band structure analysis shown in Fig. 4 is based on an infinite SSH stripe, the edge modes still preserve in a finite two-boson synthetic lattice [i.e., a square-shaped 2D SSH panel as in Fig. 3(b)], which we demonstrate with numerical simulations in Fig. 5. In simulations, we consider a 1D SSH lattice with 62 lattice sites (31 unit cells), i.e., 
$-15 \leqslant k \leqslant 15$ in Fig. 3(a). It gives a two-boson synthetic lattice with $62 \times 62$ sites in two dimensions. For the convenience of plotting in Fig. 5, lattice site $C_{k}$ in Fig. 3(a) is labeled as the $2 k$-th site (i.e., $C_{k}$ sites have indices $2 k=-30,-28, \ldots, 30$ ) and $D_{k}$ as the $(2 k+1)$-th site (i.e., $D_{k}$ sites have indices $2 k+1=-29,-27, \ldots, 31)$. We numerically solve Eq. (21) together with the input-output formalism following the procedures described in Sec. II. The probability amplitude that two bosons are respectively observed on lattice sites $C_{m}$ and $C_{n}\left(D_{m}\right.$ and $D_{n}$, and $C_{m}$ and $\left.D_{n}\right)$ in Fig. 3(a) is

$$
\begin{aligned}
& \left(C_{m}, C_{n}\right): \lambda_{m n}(t) \\
& \quad=\sqrt{2-\delta_{m n}} v_{2 m, 2 n}(-15 \leqslant m \leqslant n \leqslant 15), \\
& \left(D_{m}, D_{n}\right): \mu_{m n}(t) \\
& \quad=\sqrt{2-\delta_{m n}} v_{2 m+1,2 n+1}(-15 \leqslant m \leqslant n \leqslant 15), \\
& \left(C_{m}, D_{n}\right): \xi_{m n}(t) \\
& \quad=\sqrt{2} v_{2 m, 2 n+1}(-15 \leqslant m, n \leqslant 15),
\end{aligned}
$$

where $\delta_{m n}$ is the Kronecker delta function, and the average boson number on each site of the 1D SSH lattice can be calculated as

$$
\begin{aligned}
\text { Site } C_{k}: N_{2 k}(t)= & \sum_{j=-15}^{k-1}\left|\lambda_{j k}(t)\right|^{2}+2\left|\lambda_{k k}(t)\right|^{2} \\
& +\sum_{j=k+1}^{15}\left|\lambda_{k j}(t)\right|^{2}+\sum_{j=-15}^{15}\left|\xi_{k j}(t)\right|^{2}, \\
\text { Site } D_{k}: N_{2 k+1}(t)= & \sum_{j=-15}^{k-1}\left|\mu_{j k}(t)\right|^{2}+2\left|\mu_{k k}(t)\right|^{2} \\
& +\sum_{j=k+1}^{15}\left|\mu_{k j}(t)\right|^{2}+\sum_{j=-15}^{15}\left|\xi_{j k}(t)\right|^{2} .
\end{aligned}
$$

We plot evolutions of the average boson numbers on the 1D SSH lattice in Figs. 5(a), 5(c), and 5(e), while plotting the corresponding distributions of the excitation probabilities on the two-boson synthetic lattice in Figs. 5(b), 5(d), and 5(f), respectively. In Figs. 5(a) and 5(b), we perform the simulation in the nontrivial phase and apply the external source in Eq. (16) with $\Delta \varepsilon=3.16 \mathrm{~g}$ to excite two bosons on lattice sites $C_{0}$ and $D_{15}$ (i.e., the 0 th and 31 st sites in the $1 \mathrm{D}$ lattice), which corresponds to the excitation at the site $(0,31)$ in the twoboson synthetic lattice in Fig. 5(b). One clearly sees the edge modes in the two-boson synthetic lattice in Fig. 5(b), which features bidirectional propagation because the corresponding modes $A$ (with a positive slope) and $A^{\prime}$ (with a negative slope) in Fig. 4(b) are both excited by the source. The corresponding evolution of the average boson numbers in the 1D lattice in Fig. 5(a) exhibits the same feature, where one boson excited at the boundary is localized at $D_{15}$, while the other boson excited at $C_{0}$ propagates along both directions in one dimension. The band analysis based on the two-boson synthetic lattice is therefore useful for us to understand the evolution of the boson number distribution along the 1D lattice. As a comparison, if we excite the bulk site $(0,0)$ in the nontrivial phase [Figs. 5(c) and 5(d)], or excite the edge site $(0,31)$ in
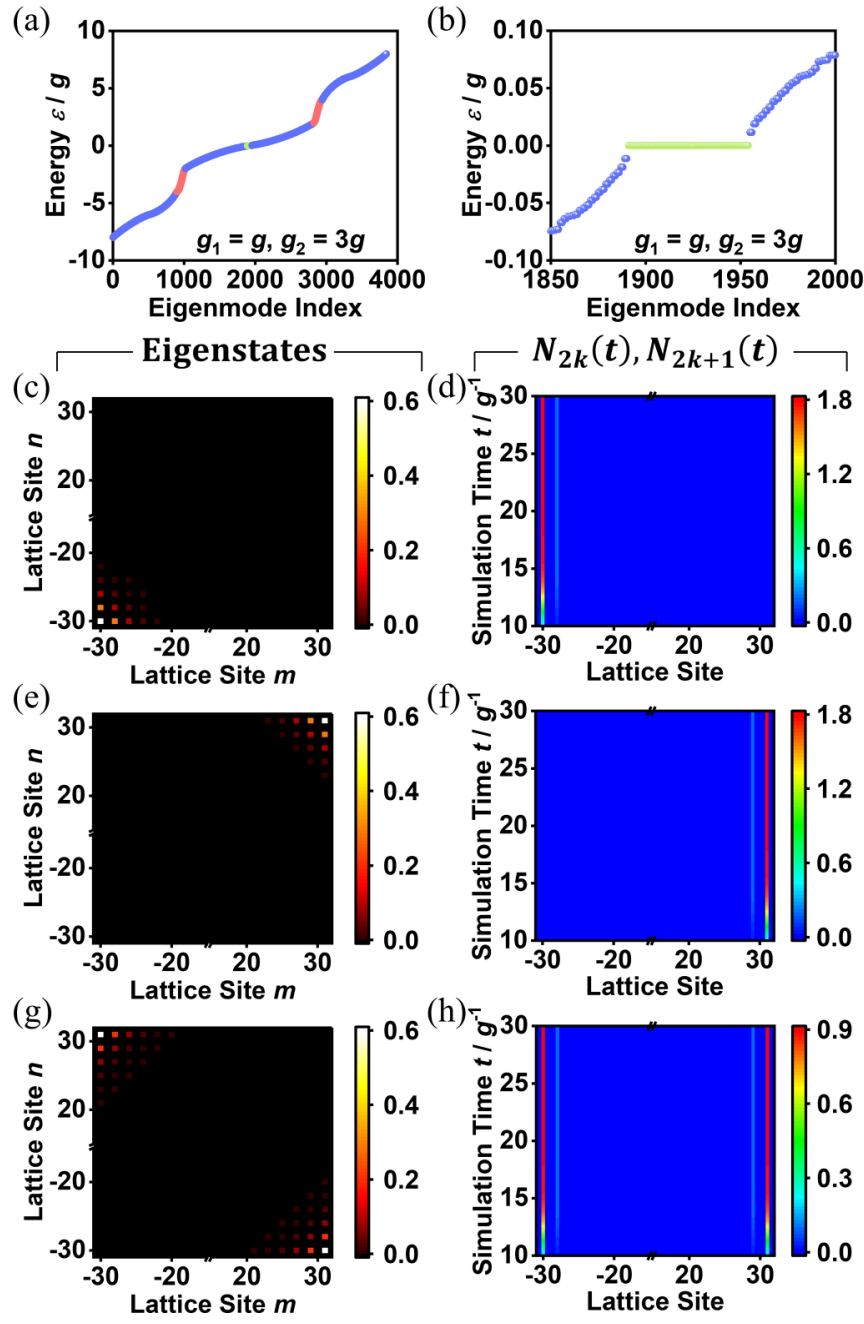

FIG. 6. Two-boson corner modes in the two-boson SSH synthetic lattice without interactions. (a) Eigenenergies of an SSH panel $(-15 \leqslant m, n \leqslant 15)$ with coupling strengths $g_{1}=g, g_{2}=3 g$. Bulk modes are colored blue, edge modes are colored red, and modes with zero energy are colored green. (b) Zoom-in plotting of (a). (c), (e), (g) Eigenstate distributions of zero-energy corner modes on an $\mathrm{SSH}$ panel $(-15 \leqslant m, n \leqslant 15)$ with coupling strengths $g_{1}=g, g_{2}=3 g$. Eigenstates are localized at (c) site $C_{-15}$, (e) site $D_{15}$, and (g) sites $C_{-15}$ and $D_{15}$. (d), (f), (h) Average boson number simulation results of two-boson corner modes, where (d) two bosons on site $C_{-15}$, (f) two bosons on site $D_{15}$, (h) one boson on site $C_{-15}$, and the other on $D_{15}$ are excited. $\Delta \varepsilon=0, t_{0}=10 g^{-1}$ and $\tau^{2}=10 g^{-2}$ in (d), (f), (h).

the trivial phase [Figs. 5(e) and 5(f)] in simulations, no edge modes are observed.

We shall clarify that, as mentioned in Eq. (23), without boson-boson interaction, the two-boson dynamics on a 1D SSH lattice can be readily inferred from the single-boson dynamics on a 1D SSH lattice. For example, in Fig. 5(a), the two-boson edge mode is in fact one boson localized at the boundary of the original 1D SSH chain and the other propagating inside the 1D chain. In another example of Fig. 6 , our two-boson synthetic lattice shows that a two-boson corner mode is simply the combination of two single-boson boundary modes, where both bosons are localized on either terminal of the $1 \mathrm{D} \mathrm{SSH}$ chain in the nontrivial phase. 
In this section, we utilize a simple example to show that a perspective from a synthetic lattice helps one to understand the $N$-boson dynamics in a 1D lattice. This capability will be much more important when the interaction between bosons is introduced, which we will show in the next two sections.

\section{TWO BOSONS ON AN SSH LATTICE WITH INTERACTIONS}

An interacting quantum system certainly shows a far richer physical behavior as compared with a noninteracting systems. The study of the $N$-boson synthetic lattice provides a unique perspective to explore multiboson problems with interactions. As an illustration, we consider two bosons on the same 1D SSH lattice as that in Sec.III, but with boson-boson interactions of the extended Bose-Hubbard type. The corresponding Hamiltonian is described by

$$
\begin{aligned}
H= & \sum_{k=-\infty}^{+\infty}\left(g_{1} c_{k}^{\dagger} d_{k}+g_{2} c_{k+1}^{\dagger} d_{k}+\text { H.c. }\right) \\
& +\frac{U}{2} \sum_{k=-\infty}^{+\infty} \sum_{s=-R}^{+R}\left(c_{k+s}^{\dagger} c_{k}^{\dagger} c_{k} c_{k+s}\right. \\
& \left.+d_{k+s}^{\dagger} d_{k}^{\dagger} d_{k} d_{k+s}+2 c_{k+s}^{\dagger} d_{k}^{\dagger} d_{k} c_{k+s}\right),
\end{aligned}
$$

where $U$ is the interaction strength and $R$ is the interaction range. Different from previous works [38-41], here we show the capability of our method to understand the multiboson problem with long-range nonlocal interactions, and predict the existence of topological states that have not been explored before. For this purpose, we take the same two-boson state in Eq. (19) and apply the Schrödinger equation with the Hamiltonian in Eq. (27) (assuming $R \geqslant 2$ ), and obtain

$$
\begin{aligned}
i\left(\dot{v}_{2 m, 2 n}+\dot{v}_{2 n, 2 m}\right) & \\
= & g_{1}\left(v_{2 m, 2 n+1}+v_{2 n, 2 m+1}+v_{2 m+1,2 n}+v_{2 n+1,2 m}\right) \\
& +g_{2}\left(v_{2 m, 2 n-1}+v_{2 n, 2 m-1}+v_{2 m-1,2 n}+v_{2 n-1,2 m}\right) \\
& +U \delta_{|m-n| \leqslant R}\left(v_{2 m, 2 n}+v_{2 n, 2 m}\right), \\
i\left(\dot{v}_{2 m+1,2 n+1}+\dot{v}_{2 n+1,2 m+1}\right) & \\
= & g_{1}\left(v_{2 m, 2 n+1}+v_{2 n, 2 m+1}+v_{2 m+1,2 n}+v_{2 n+1,2 m}\right) \\
& +g_{2}\left(v_{2 m+2,2 n+1}+v_{2 n+2,2 m+1}+v_{2 m+1,2 n+2}\right. \\
& \left.+v_{2 n+1,2 m+2}\right)+U \delta_{|m-n| \leqslant R}\left(v_{2 m+1,2 n+1}+v_{2 n+1,2 m+1}\right), \\
i\left(\dot{v}_{2 m, 2 n+1}+\dot{v}_{2 n+1,2 m}\right) & \\
= & g_{1}\left(v_{2 m, 2 n}+v_{2 n, 2 m}+v_{2 m+1,2 n+1}+v_{2 n+1,2 m+1}\right) \\
& +g_{2}\left(v_{2 m, 2 n+2}+v_{2 n+2,2 m}+v_{2 m-1,2 n+1}\right. \\
& \left.+v_{2 n+1,2 m-1}\right)+U \delta_{|m-n| \leqslant R}\left(v_{2 m, 2 n+1}+v_{2 n+1,2 m}\right), \quad(28
\end{aligned}
$$

where $\delta_{|m-n| \leqslant R}=1$ if $|m-n| \leqslant R$ and $=0$ otherwise. Imposing the exchange symmetry on the wave function $\left(v_{m n}=v_{n m}\right)$, we can rewrite Eq. (28) as

$$
\begin{aligned}
i \dot{v}_{2 m, 2 n}= & g_{1}\left(v_{2 m, 2 n+1}+v_{2 m+1,2 n}\right) \\
& +g_{2}\left(v_{2 m, 2 n-1}+v_{2 m-1,2 n}\right) \\
& +U \delta_{|m-n| \leqslant R} v_{2 m, 2 n},
\end{aligned}
$$

(a)

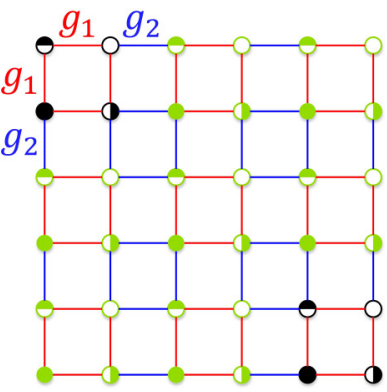

- $C_{m} C_{n}, \lambda_{m n}, v_{2 m, 2 n}$

$\ominus C_{m} D_{n}, \xi_{m n}, v_{2 m, 2 n+1}$

- $D_{m} C_{n}, \xi_{n m}, v_{2 m+1,2 n}$

○ $D_{m} D_{n}, \mu_{m n}, v_{2 m+1,2 n+1}$
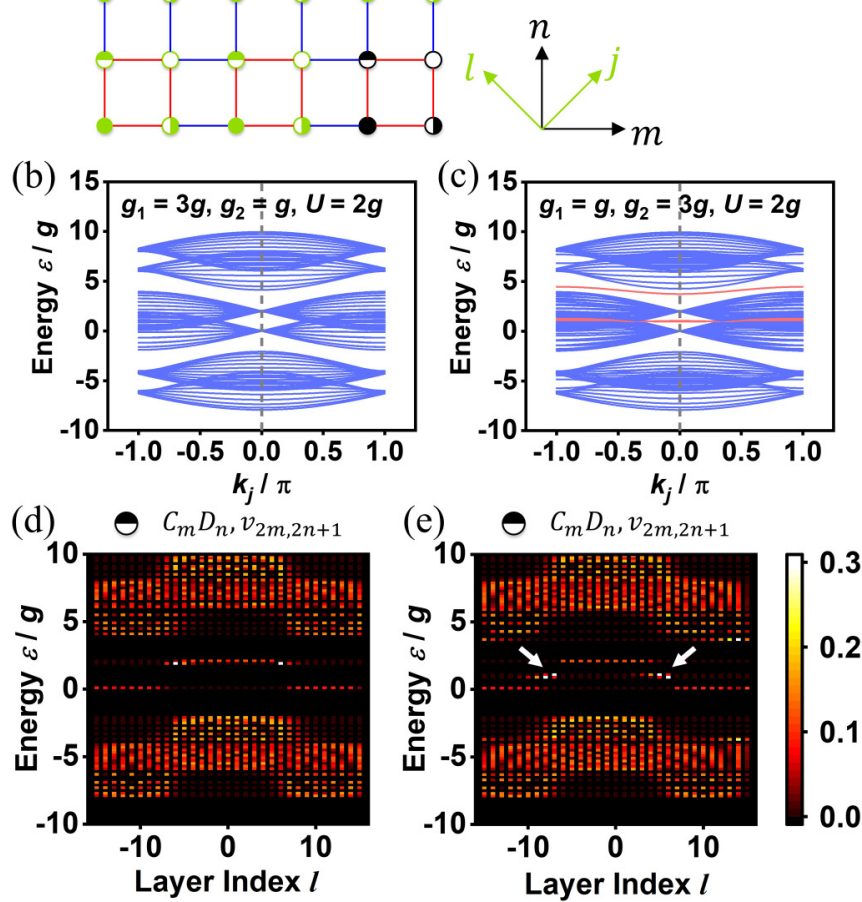

FIG. 7. The two-boson SSH synthetic lattice with interactions. (a) The two-boson synthetic lattice with the exchange symmetry restriction on the wave function, where green spots indicate nonzero on-site potentials. (b), (c) Projected band structures of the lattice in (a) with $U=2 g,\left(g_{1}, g_{2}\right)=(3 g, g)$, or $(g, 3 g)$. (d), (e) Eigenstate distributions of $C_{m} D_{n}$-type lattice sites corresponding to the band structures in (b), (c) at $k_{j}=0$ [gray dashes in (b), (c)], in topologically (d) trivial and (e) nontrivial phases. Interface modes are indicated by white arrows. $R=6$ and $-15 \leqslant l \leqslant 15$ in (b)-(e).

$$
\begin{aligned}
i \dot{v}_{2 m, 2 n+1}= & g_{1}\left(v_{2 m, 2 n}+v_{2 m+1,2 n+1}\right) \\
& +g_{2}\left(v_{2 m, 2 n+2}+v_{2 m-1,2 n+1}\right) \\
& +U \delta_{|m-n| \leqslant R} v_{2 m, 2 n+1}, \\
i \dot{v}_{2 m+1,2 n}= & g_{1}\left(v_{2 m, 2 n}+v_{2 m+1,2 n+1}\right) \\
& +g_{2}\left(v_{2 m+2,2 n}+v_{2 m+1,2 n-1}\right) \\
& +U \delta_{|m-n| \leqslant R} v_{2 m+1,2 n}, \\
i \dot{v}_{2 m+1,2 n+1}= & g_{1}\left(v_{2 m, 2 n+1}+v_{2 m+1,2 n}\right) \\
& +g_{2}\left(v_{2 m+2,2 n+1}+v_{2 m+1,2 n+2}\right) \\
& +U \delta_{|m-n| \leqslant R} v_{2 m+1,2 n+1} .
\end{aligned}
$$

Equation (29) therefore again can be mapped to a synthetic lattice in two dimensions as shown in Fig. 7(a). Here $U$ appears as the on-site potential on the diagonal with a width of $2 R+1$ in this two-boson synthetic lattice, as a result of the nonlinear interaction terms in Eq. (27), as indicated by the green-colored lattice sites in Fig. 7(a). These lattice sites with nonzero on-site potentials compose the "nonlinear lattice 
region," while the sites without on-site potentials compose the "linear lattice region." Figure 7(a) features translational symmetry along the $j$ axis $[\hat{\mathbf{j}}=(\hat{\mathbf{m}}+\hat{\mathbf{n}}) / \sqrt{2}]$, and the corresponding wave vector $k_{j}$ is in fact the quasimomentum of the motion of the center of mass of these two bosons $\left(k_{j}=\right.$ $\left.k_{m}+k_{n}\right)$ [31].

For a stripe with finite width along the $l$ axis $[\hat{\mathbf{I}}=(\hat{\mathbf{n}}-$ $\hat{\mathbf{m}}) / \sqrt{2}$, perpendicular to the $j$ axis] and infinite length along the $j$ axis, we perform a Fourier transform along the $j$ axis and then plot the projected band structure $\varepsilon\left(k_{j}\right)$ with different combinations of parameters $g_{1}, g_{2}$, and $U$. When $U=0, g_{1}=$ $3 g$, and $g_{2}=g$, the projected band structure can be viewed as the 2D SSH band structure after projection along the $\Gamma-M$ direction in the first Brillouin zone and then band folding [see Figs. 8(c) and 8(d)]. Then we plot the projected band structure for the case $U=2 g, R=6, g_{1}=3 g$, and $g_{2}=g$ (trivial phase) in Fig. 7(b). One can see that, compared to the plot in Fig. 8(d), some of the bands inside the quasicontinuum are lifted by $2 g$, which is equal to the on-site potential $U$. In other words, $U$ shifts the potential energy of the sites in the nonlinear region. This observation is consistent with the results in Appendix B; i.e., the lifted bands with higher energies correspond to two-boson bound states (doublons) in the nonlinear lattice region around $l=0$, and the unlifted bands with lower energies correspond to free-particle scattering states in the linear region. When $U=2 g, R=6, g_{1}=g$, and $g_{2}=3 g$ (nontrivial phase), we plot the band structure in Fig. 7(c) and can see topological effects. The edge mode in the upper band gap is identified and indicated in red, yet the edge mode in the lower band gap is overlapped by lifted bulk bands (i.e., the edge mode "collapses" into scattering bulk modes). Moreover, there are other isolated bands found around $k_{j}=0$ and $\varepsilon=g$. We plot the eigenstate distributions of $C_{m} D_{n}$ sites with $k_{j}=0$ for the trivial and nontrivial phases in Figs. 7(d) and 7(e), respectively. (Other eigenstate distributions of $C_{m} C_{n}, D_{m} C_{n}$, and $D_{m} D_{n}$ sites can be found in Fig. 9.) One can see that, for the nontrivial case, there exists a pair of localized states near $\varepsilon=$ $g$ at the interface between linear and nonlinear lattice regions. These states are the interface modes. Here the interaction range $R$ must be large enough so that the isolated bands of interface modes exist and the modes at the two interfaces do not hybridize.

To see details of the band structure in Fig. 7(c), we focus on the region around $k_{j}=0$ and $\varepsilon=g$ in Fig. 10(a). One can see that these interface modes are composed of two groups of degenerate bands (bands 1 and 2, and bands 3 and 4) with slightly different energies. Figures $10(\mathrm{~b})-10(\mathrm{e})$ plot the eigenstate distributions of the corresponding four bands, respectively, in a two-boson synthetic lattice with parameters $-2 \leqslant m, n \leqslant 3$, and $R=2$ for the illustration purpose. The lower-energy group of bands corresponds to the modes on the linear side of the interface [bands 1 and 2 in Figs. 10(b) and $10(\mathrm{c})$ ], while the higher-energy group corresponds to the modes on the nonlinear side of the interface [bands 3 and 4 in Figs. 10(d) and 10(e)]. It is shown that, of the two degenerate bands in each group, one band is symmetric with respect to the line $m=n$ [Figs. 10(c) and 10(e)] and the other one is antisymmetric [Figs. 10(b) and 10(d)]. However, only the symmetric modes can be physically excited because of our exchange symmetry restriction of the wave function. The pair (a)

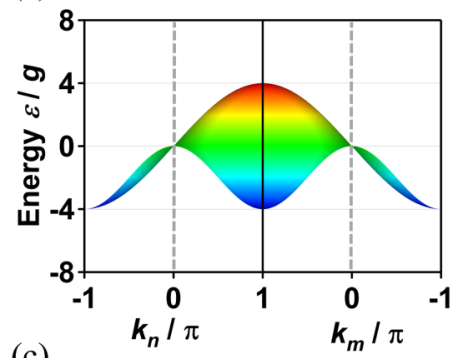

(c)

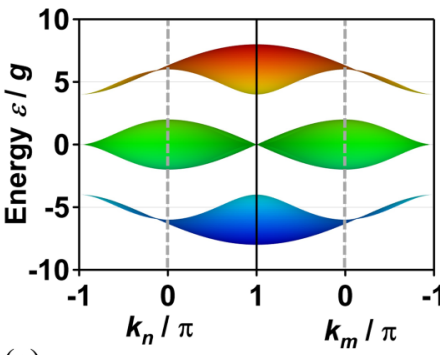

(e)

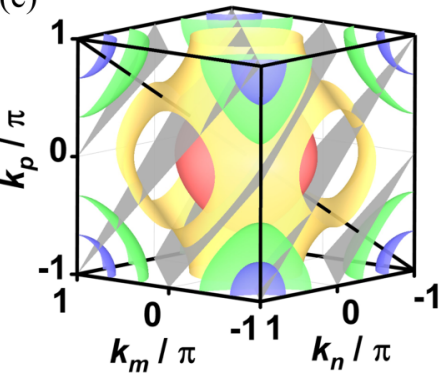

(b)

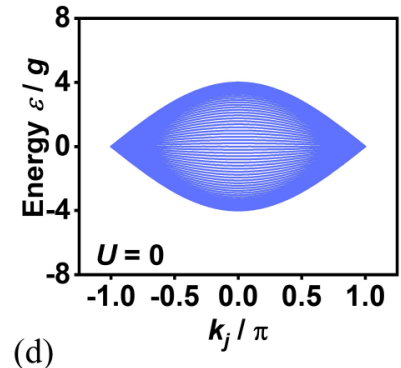

(d)

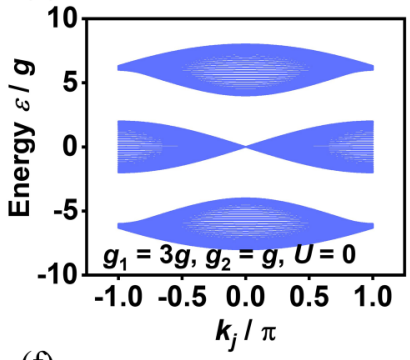

(f)

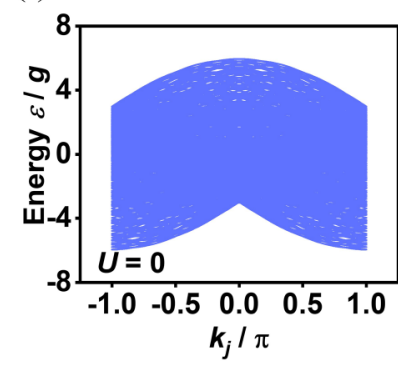

FIG. 8. Projection of band structures of multiboson synthetic lattices. (a) A side view of 2D band structure of the 2D tight-binding lattice without interactions. (b) Projected band structure of 2D tightbinding lattice when $U=0$, which can be acquired from (a) by band folding $\left(k_{j} \bmod 2 \pi\right)$. (c) A side view of 2D band structure of the 2D SSH lattice without interactions. (d) Projected band structure of 2D SSH lattice when $U=0$, which can be acquired from (c) by band folding $\left(k_{j} \bmod 2 \pi\right)$. (e) Energy isosurfaces of 3D band structure of the $3 \mathrm{D}$ tight-binding lattice without interactions. Blue: $\varepsilon=-5 g$; green: $\varepsilon=-3 g$; yellow: $\varepsilon=0$; red: $\varepsilon=3 g$; gray surfaces: $k_{j}=k_{m}+k_{n}+k_{p}=-2 \pi, \pi, 0, \pi, 2 \pi$. Plane $k_{m}+k_{n}+k_{p}=$ $\pm \pi\left(k_{m}+k_{n}+k_{p}= \pm 2 \pi\right)$ is tangential to isosurface $\varepsilon=3 g(\varepsilon=$ $-3 g)$ at $k_{m}=k_{n}=k_{p}= \pm \pi / 3\left(k_{m}=k_{n}=k_{p}= \pm 2 \pi / 3\right)$. (f) Projected band structure of 3D tight-binding lattice when $U=0$, which can be acquired from (e) by projection onto the black solid line $k_{m}=k_{n}=k_{p}$ and then band folding $\left(k_{j} \bmod 2 \pi\right)$.

of antisymmetric interface modes (bands 1 and 3 ) is an artifact produced from the 1D-2D mapping method, and does not physically exist.

We next perform numerical simulations to validate this interface mode mentioned above. Equation (29) is solved with $R=6$, and the evolutions of the average boson number distribution along the 1D SSH chain are calculated by Eqs. (24)-(26). We first consider the case that sites $C_{0}$ and $D_{6}$ are excited by a pair of separate bosons at $\Delta \varepsilon=g$ and the lattice is in the nontrivial, nonlinear phase $\left(g_{1}=g, g_{2}=3 g\right.$, $U=2 g$ ). In the corresponding plot of the evolution of the average boson number distribution in Fig. 11(a), one can see that the interface mode is excited and preserved throughout 

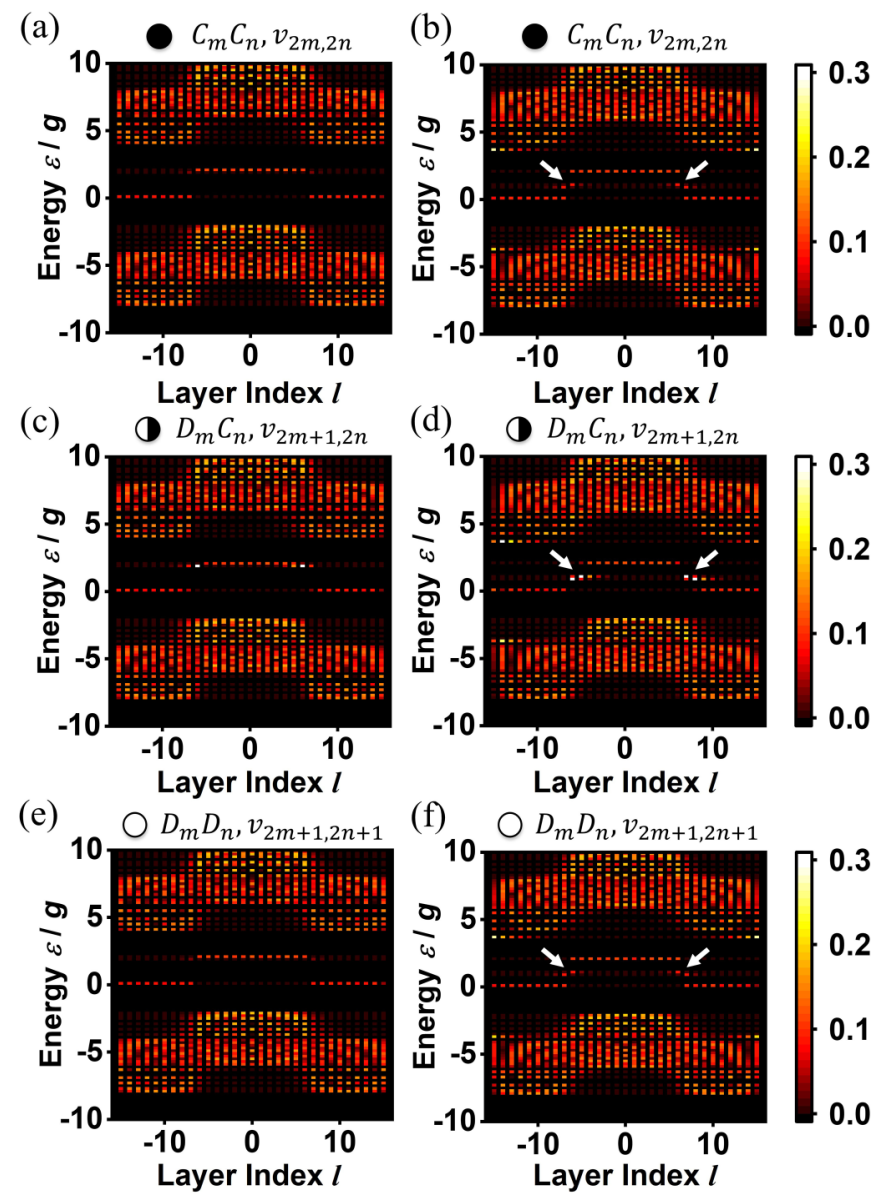

FIG. 9. Eigenstate distributions of interface modes in the twoboson SSH synthetic lattice with interactions. (a)-(f) Eigenstate distributions of $C_{m} C_{n^{-}}, D_{m} C_{n^{-}}$, and $D_{m} D_{n}$-type lattice sites corresponding to band structures in Figs. 7(b) and 7(c) at $k_{j}=0$ [gray dashes in Figs. 7(b) and 7(c)], in topologically (a), (c), (e) trivial and (b), (d), (f) nontrivial phases. Interface modes are indicated by white arrows. $R=6$ and $-15 \leqslant l \leqslant 15$ in this figure.

the simulation, as the distance between the two bosons keeps stationary. The boson scattering in Fig. 11(a) is negligible because the bands of the interface modes are relatively flat, so the group velocity of the wave packet is small. In Fig. 11(b), we take the case that $C_{0}$ and $D_{6}$ are excited but the lattice is in the trivial, nonlinear phase $\left(g_{1}=3 g, g_{2}=g, U=2 g\right)$. There is no interface mode observed. In Fig. 11(c), the lattice is in the nontrivial, nonlinear phase, but two bosons are excited simultaneously on site $C_{0}$, and thus bulk modes are excited instead. Finally, in Fig. 11(d), $C_{0}$ and $D_{6}$ are excited and the lattice is in the nontrivial phase but $U=0$, and no interface mode is found as expected. The simulations here prove that, for the Hamiltonian in Eq. (27), the nonzero on-site potential $U$, the topologically nontrivial phase $\left(g_{2}>g_{1}\right)$, and an initial excitation on the linear-nonlinear interface are all necessary conditions to successfully excite an interface mode in Fig. 7(c).

The successful demonstration of the interface modes exhibits the capability of our approach, with the two-boson synthetic lattice and the band structure analysis, to predict exotic physical phenomena. We also note that several previ- ous studies used the modified Bethe ansatz and gave explicit solutions to some specific Hamiltonians with short-range interactions [32,35,37-39,42], yet these methods still face challenges when the interactions are arbitrarily nonlocal $(R \geqslant$ $2)$ or the total boson number is large $(N \geqslant 3)$. Our theoretical approach proposed here is more general and, as we show in Secs. IV and $\mathrm{V}$, is able to deal with multiboson dynamics with long-range interactions and discover exotic states in these systems.

\section{THREE BOSONS ON A TIGHT-BINDING LATTICE WITH INTERACTIONS}

We have discussed the 2D synthetic SSH lattice induced by the two-boson physics in Secs. III and IV. In this section, we demonstrate a three-dimensional (3D), three-boson synthetic lattice, which is constructed by three indistinguishable bosons on a 1D tight-binding extended Bose-Hubbard Hamiltonian, and show that it possesses exotic trimer states.

We consider a Hamiltonian which goes beyond previous studies [63-65] with nonlocal interactions:

$$
H=g \sum_{k=-\infty}^{+\infty}\left(b_{k}^{\dagger} b_{k+1}+b_{k+1}^{\dagger} b_{k}\right)+\frac{U}{2} \sum_{k=-\infty}^{+\infty} \sum_{s=-R}^{R} b_{k+s}^{\dagger} b_{k}^{\dagger} b_{k} b_{k+s},
$$

and the three-boson state

$$
|\phi(t)\rangle=\frac{1}{\sqrt{6}} \sum_{m=-\infty}^{+\infty} \sum_{n=-\infty}^{+\infty} \sum_{p=-\infty}^{+\infty} v_{m n p}(t) b_{m}^{\dagger} b_{n}^{\dagger} b_{p}^{\dagger}|0\rangle .
$$

By substituting Eqs. (30) and (31) into the Schrödinger equation, we get the differential equations for the $v_{m n p}$ 's $(R \geqslant$ 2):

$$
\begin{aligned}
i \sum_{\text {permu }} \dot{v}_{m n p}= & \sum_{\text {permu }}\left[g \left(v_{m-1, n, p}+v_{m+1, n, p}+v_{m, n-1, p}\right.\right. \\
& \left.+v_{m, n+1, p}+v_{m, n, p-1}+v_{m, n, p+1}\right) \\
& \left.+\left(\delta_{|m-n| \leqslant R}+\delta_{|n-p| \leqslant R}+\delta_{|p-m| \leqslant R}\right) U v_{m n p}\right] .
\end{aligned}
$$

For this three-boson case, the wave function exchange symmetry is defined as $v_{m n p}=v_{m p n}=v_{n m p}=v_{n p m}=v_{p m n}=$ $v_{p n m}$, and then the permutation sum in Eq. (32) can be removed:

$$
\begin{aligned}
i \dot{v}_{m n p}= & g\left(v_{m-1, n, p}+v_{m+1, n, p}+v_{m, n-1, p}+v_{m, n+1, p}\right. \\
& \left.+v_{m, n, p-1}+v_{m, n, p+1}\right)+\left(\delta_{|m-n| \leqslant R}+\delta_{|n-p| \leqslant R}\right. \\
& \left.+\delta_{|p-m| \leqslant R}\right) U v_{m n p} .
\end{aligned}
$$

The first six terms in Eq. (33) correspond to a 3D tightbinding lattice, which spans the full 3D $(m, n, p)$ space with equal coupling strengths $g$, as is shown by the cubic structure in Fig. 12(a). (If we do not assume the exchange symmetry, and instead restrict that $v_{m n p}=0$ except in the region $m \leqslant n \leqslant p$, Eq. (32) would be translated to the tetrahedron structure in Fig. 13(c), which does not support the following band structure analysis.) After ignoring the nonlinearity for 
(a)

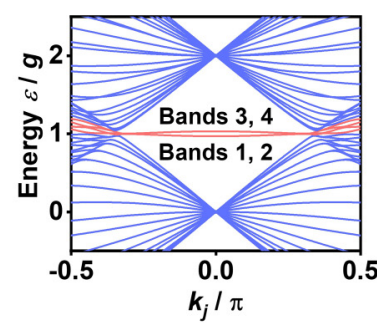

(b)

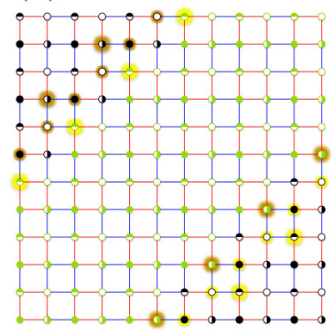

(c)

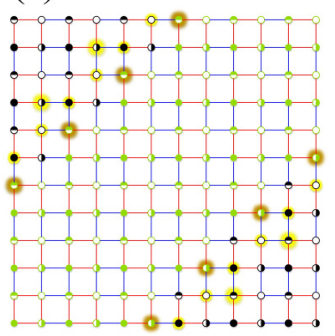

(d)

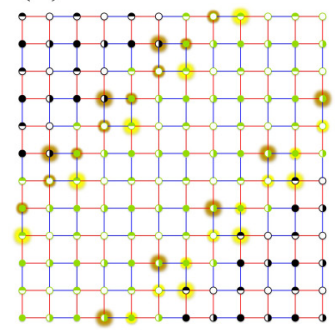

(e)

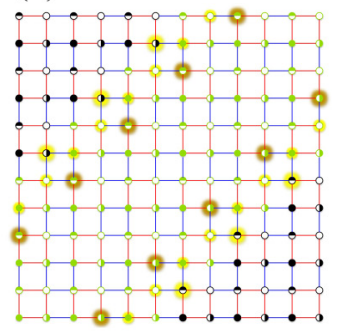

FIG. 10. Eigenstate distributions of interface modes in the two-boson SSH synthetic lattice with interactions. (a) Zoom-in view of the projected band structure Fig. 7(c), with $g_{1}=g, g_{2}=3 g, U=2 g, R=6$, and $-15 \leqslant l \leqslant 15$. (b)-(e) Eigenstate distributions of (b) band 1 (antisymmetric band with lower energy), (c) band 2 (symmetric band with lower energy), (d) band 3 (antisymmetric band with higher energy), and (e) band 4 (symmetric band with higher energy) at $k_{j}=0$. The size of the yellowish glow around lattice sites qualitatively represents the intensity of the eigenstate on the lattice sites, and the brightness (light yellow or dark yellow) represents the plus (light) or minus (dark) sign of the eigenstate intensity. $-2 \leqslant m, n \leqslant 3$ and $R=2$ in (b)-(e) for illustration purposes.

the moment (i.e., $U=0$ ), the band structure of the cubic three-boson synthetic lattice is

$$
\varepsilon_{\text {tight-binding }}^{3 \mathrm{D}}\left(k_{m}, k_{n}, k_{p}\right)=2 g\left(\cos k_{m}+\cos k_{n}+\cos k_{p}\right) \text {, }
$$

which can be shown by the energy isosurfaces in Fig. 12(b). At the center point of the cubic reciprocal lattice ( $\Gamma$ point), $\varepsilon$ takes its maximum value of $6 g$, while at the corners $(R$ point), $\varepsilon$ takes the minimum value of $-6 g$. Now, we consider the added nonzero on-site potential $U$ on lattice sites around the line $m=n=p$ [the $j$ axis, $\hat{\mathbf{j}}=(\hat{\mathbf{m}}+\hat{\mathbf{n}}+\hat{\mathbf{p}}) / \sqrt{3}$ ], as indicated by the green spots in Fig. 12(a). One can perform Fourier transform along the $j$ axis and obtain the projected
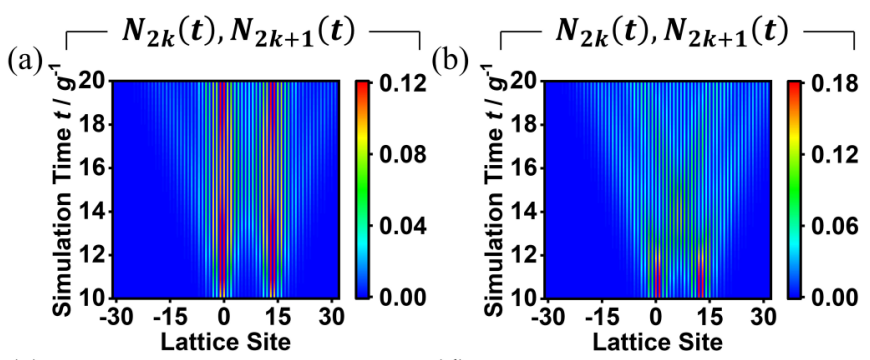

(c)

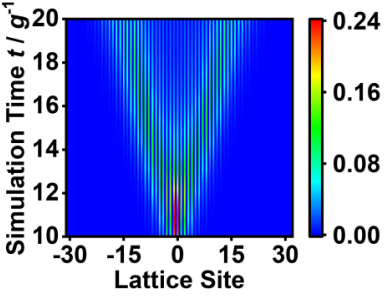

(d)

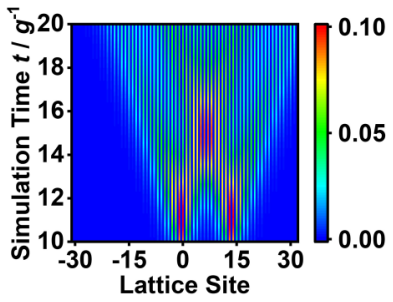

FIG. 11. Average boson number simulation results of interface modes in the two-boson SSH synthetic lattice with interactions. (a) The wave packet evolution after exciting two bosons on sites $C_{0}$ and $D_{6}$, respectively, with $U=2 g, g_{1}=g, g_{2}=3 g$, and $\Delta \varepsilon=g$. (b) The wave packet evolution after exciting two bosons on sites $C_{0}$ and $D_{6}$, respectively, with $U=2 g, g_{1}=3 g, g_{2}=g$, and $\Delta \varepsilon=g$. (c) The wave packet evolution after exciting two bosons on site $C_{0}$ simultaneously, with $U=2 g, g_{1}=g, g_{2}=3 g$, and $\Delta \varepsilon=g$. (d) The wave packet evolution after exciting two bosons on sites $C_{0}$ and $D_{6}$, respectively, with $U=0, g_{1}=g, g_{2}=3 g$, and $\Delta \varepsilon=0 . R=6$, $t_{0}=10 g^{-1}$, and $\tau^{2}=10 g^{-2}$ in this figure. band structure $\varepsilon\left(k_{j}\right)$. Here $k_{j}=k_{m}+k_{n}+k_{p}$. For the linear case with $U=0, \varepsilon\left(k_{j}\right)$ can be understood from Eq. (34) by a projection onto the $\Gamma-R$ direction and then band folding into the first Brillouin zone [see Figs. 8(e) and 8(f)].

We then consider the strong-interaction case with $U=12 \mathrm{~g}$ and plot the projected band structure $\varepsilon\left(k_{j}\right)$ in Fig. 14(a). One can see that there are four separate quasicontinuum bands. We find that these bands correspond to different multiboson states when we plot the eigenstates of these four quasicontinuum bands in the $l_{1}-l_{2}$ plane, as shown by the diagram in Fig. $14(b)$. Here $\hat{\mathbf{l}}_{1}=(\hat{\mathbf{n}}-\hat{\mathbf{m}}) / \sqrt{2}$ and $\hat{\mathbf{l}}_{2}=(\hat{\mathbf{p}}-\hat{\mathbf{m}}) / \sqrt{2}$ are two linearly independent directions orthogonal to the $j$ axis in the $3 \mathrm{D}$ space, and $\left|l_{1}\right|,\left|l_{2}\right|,\left|l_{1}-l_{2}\right|$ are actually the relative distances between each two of these three bosons. The $l_{1}-l_{2}$ plane is divided into 19 regions by six lines $\left|l_{1}\right|=$ $\pm R,\left|l_{2}\right|= \pm R,\left|l_{1}-l_{2}\right|= \pm R$, classified into four categories, which are dominated by eigenstates from different bands. In the blue regions as shown in Fig. 14(b), we find that they correspond to the band with the lowest energy in Fig. 14(a), as confirmed by the eigenstate distribution at $\left(k_{j}, \varepsilon\right)=(0,5.62 g)$ shown in Fig. 14(c). The areas of the blue regions are infinitely large if $-\infty<l_{1}, l_{2}<+\infty$, and none of the three $\delta$ functions in Eq. (33) is equal to 1 in these regions. It should also be (a)

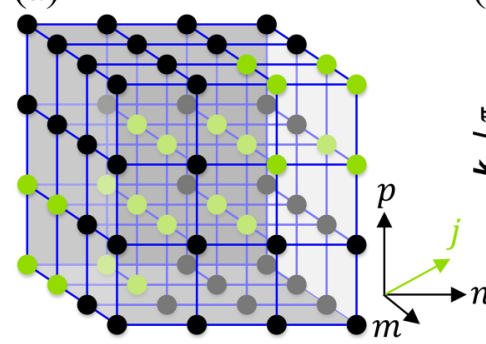

(b)

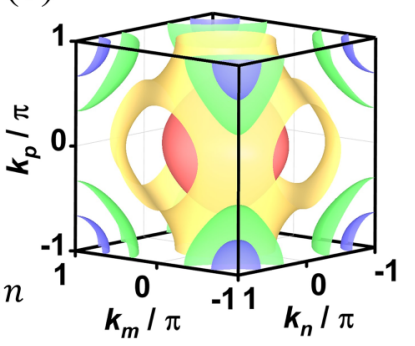

FIG. 12. The three-boson tight-binding synthetic lattice with interactions. (a) The three-boson synthetic lattice with the exchange symmetry restriction on the wave function $v_{m n p}(t)$, where green spots indicate nonzero on-site potentials. (b) Energy isosurface of the 3D band structure of the lattice in (a) in the first Brillouin zone when $U=0$. Red: $\varepsilon=3 g$; yellow: $\varepsilon=0$; green: $\varepsilon=-3 g$; blue: $\varepsilon=-5 g$. 


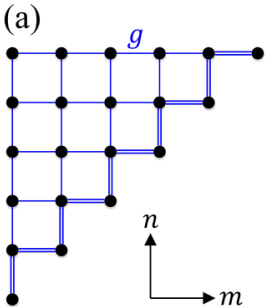

(b)

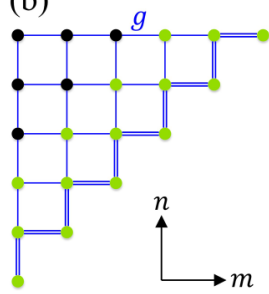

(c)

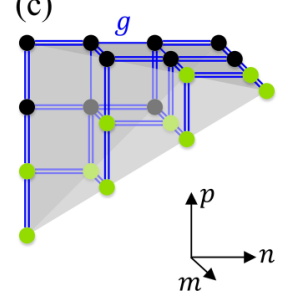

FIG. 13. The lattice structure formed by the wave function without the exchange symmetry restriction. (a) Two bosons on a 1D tight-binding lattice without interactions; $v_{m n}=0$ except in the region $m \leqslant n$. (b) Two bosons on a 1D tight-binding lattice with interactions; $v_{m n}=0$ except in the region $m \leqslant n$. (c) Three bosons on a $1 \mathrm{D}$ tight-binding lattice with interactions; $v_{m n p}=0$ except in the region $m \leqslant n \leqslant p$. Green spots indicate lattice sites with nonzero on-site potentials, and double lines indicate coupling strengths that are not equal to $g$ (the coupling strength in the $1 \mathrm{D}$ tight-binding lattice).
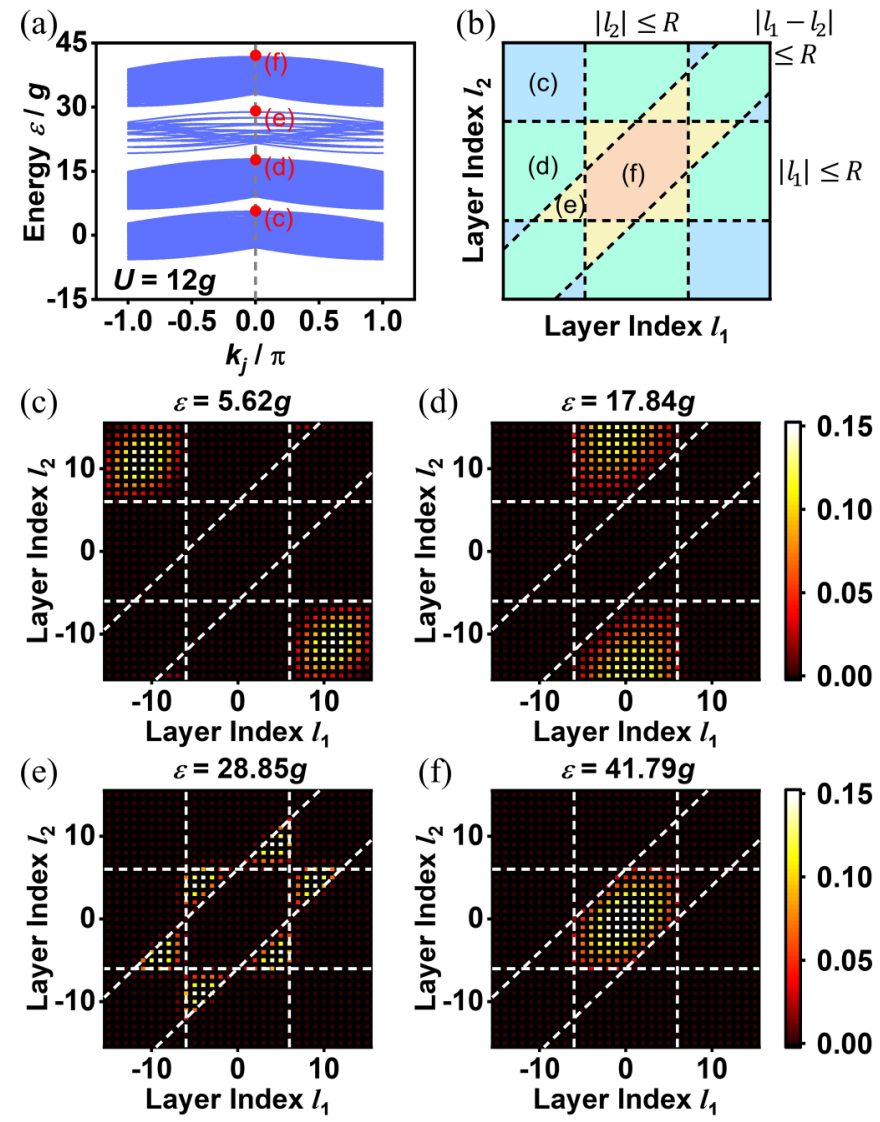

FIG. 14. Projected band structure and eigenstate distributions of the three-boson tight-binding synthetic lattice with interactions. (a) Projected band structure of the three-boson synthetic lattice with $U=12 g$. (b) Regional diagram of eigenstate distributions. Blue area: bands of lowest energy; cyan area: bands of second-lowest energy; orange area: bands of second-highest energy; red area: bands of highest energy. (c)-(f) Representative eigenstate distributions corresponding to the band structure at $k_{j}=0$ [gray dash in (a)] and at (c) $\varepsilon=5.62 \mathrm{~g}$, (d) $\varepsilon=17.84 \mathrm{~g}$, (e) $\varepsilon=28.85 \mathrm{~g}$, and (f) $\varepsilon=41.79 \mathrm{~g}$. $R=6$ and $-15 \leqslant l_{1}, l_{2} \leqslant 15$ in this figure. noted that, although in Fig. 14(c), only the left-top and rightbottom corners of the $l_{1}-l_{2}$ plane are occupied, the eigenstate distributions at other eigenenergies in the lowest-energy band fill up the rest of the blue regions in Fig. 14(b). These states are three-boson scattering states where bosons are not bound to each other and do not interact with each other via the nonlinearity. In the infinite cyan regions, one of the three $\delta$ functions in Eq. (33) is equal to 1, and these states consist of a doublon and a free particle [see the eigenstate distribution at $\left(k_{j}, \varepsilon\right)=(0,17.84 g)$ shown in Fig. 14(d)], residing in the second-lowest energy band in Fig. 14(a). Moreover, the red region denotes the tightly bound triplon states [see the eigenstate distribution at $\left(k_{j}, \varepsilon\right)=(0,41.79 g)$ shown in Fig. 14(f)] with the strongest interaction (consequently the highest energy) in Fig. 14(a). Nevertheless, the most intriguing result discovered here is the hexagramlike, weakly bound triplon state [see the eigenstate distribution at $\left(k_{j}, \varepsilon\right)=(0,28.85 g)$ shown in Fig. 14(e)] in the orange regions. Such an exotic state in Fig. 14(e) has not been thoroughly understood previously in the model without the nonlocal interaction [63]. In this state, compared to the tightly bound trimer in the red region, the relative distances between each two of these three particles cannot all be smaller than $R$, while compared to the dimer-monomer states in the cyan regions, none of the relative distances between each two of these three particles is allowed to be larger than $2 R$, albeit there is no artificial discontinuity at $2 R$ in the Hamiltonian in Eq. (30). This "virtual potential wall" at $2 R$ is entirely attributed to the boson-boson blockade effect. Therefore, in this fascinating state in Fig. 14(e), three bosons are loosely localized together, neither too close to nor too far from one another.

\section{DISCUSSIONS AND CONCLUSIONS}

In this paper, we propose a theoretical framework to treat the multiboson dynamics in a one-dimensional lattice as an $N$-boson synthetic lattice by applying exchange symmetry restrictions to the wave function. In such an artificially constructed $\mathrm{N}$-boson synthetic lattice, one can mathematically perform band structure analysis and the resulting $\mathrm{N}$-dimensional band structure provides a unique perspective to analyze the multiboson dynamics on 1D lattices. We show that, for complicated Hamiltonians with boson-boson interactions, projected band structures could be understood from the full $\mathrm{N}$-dimensional band structure, and nontrivial multiboson states (such as interface modes in Sec. IV and weakly bound triplon states in Sec. V) can be successfully predicted. In addition, the 1D lattice required in this paper to hold these multiple bosons can be in either real or synthetic dimensions per se. If this 1D lattice itself is in synthetic dimensions (e.g., formed by exploiting boson frequencies, orbital angular momenta, etc. [15]), our theory suggests that this multiboson approach might enable the construction of arbitrarily multidimensional systems on an otherwise zero-dimensional platform, together with conventional strategies of synthetic dimensions. Mathematically, if $N$ bosons are excited on a platform with $D$ real dimensions and $M$ synthetic dimensions, the system can be eventually expanded to $(D+M) N$ dimensions (with exchange symmetry restrictions). 
We also briefly suggest several possible experimental platforms for exploring $N$-boson dynamics in the 1D lattices where our proposed approach can be conducted. For lattices in the real space, coupled photonic waveguides and cavities [44,45,66-74], cold bosonic atoms in optical lattices [75-79], and superconducting circuits [80-83] are state of the art technologies for implementations. For example, the coupled waveguide array fabricated to study the two-photon quantum walks in a 1D SSH lattice [74] gives an experimental demonstration of our discussions in Sec. III. In such a photonic waveguide configuration, evanescently coupled parallel waveguides are fabricated by direct laser writing, to form a 1D lattice in the real space. The coupling strengths can be tuned by the distances between neighboring waveguides. The temporal evolution of quantum states is mapped to the light propagation along the waveguide axes ( $z$ direction), so the interactions might be simulated by changing the refractive indices of the waveguides. This can be achieved by altering the laser power during fabrication. In cold atom configurations, traps formed by an optical standing wave serve as the lattice in the real space. The coupling strengths, or the atoms' tunneling probabilities between neighboring traps, are tunable by the profile of the optical standing wave. The interactions of atoms are also more prominent than those of photons $[84,85]$, yet the experimental setups of optical lattices are less compact and may not be as amenable for miniaturization as the photonic systems because of the nano-Kelvin temperature regime required [86]. Finally, our proposed model can also find potential experiment platforms in acoustics [56,87].

As for the synthetic space, a 1D tight-binding model and SSH Hamiltonians without interactions are readily accessible via synthetic frequency dimensions in few-ring resonators with electro-optic modulation [17,58,88-90]. When the modulation frequency coincides with the free spectral range of the resonator, neighboring longitudinal modes are coupled to form a 1D lattice. For a silicon resonator with a $200 \mu \mathrm{m}$ radius operating in the telecommunication band, the modulation frequency should be $\sim 50 \mathrm{GHz}$, which is achievable using lithium niobate modulators [91,92]. Artificial boundaries of the lattices along frequency dimensions could be achieved by incorporating group velocity dispersions in ring resonators or introducing lossy absorbers at particular frequencies [71,93]. Group velocity dispersions in silicon resonators can be engineered within a broad range of values by designing the cross-sectional geometry of the waveguide [94,95], and loss mechanisms may include the absorptions of atoms [96-99], plasmons [100,101], semiconductors [102-104], and the resonances of other coupled cavities [93,105-107]. Interactive terms in Hamiltonians can be constructed from four-wavemixing processes with the nonlinear effect [108], where local (nonlocal) interactions correspond to the process of selfphase (cross-phase) modulation. In a $200 \mu \mathrm{m}$ radius silicon resonator, the coupling strengths $g$ along the frequency dimension are $\sim 1 \mathrm{GHz}$ (modulation depth divided by round-trip time). Although it is challenging to provide a comparable interaction strength $U$ in silicon resonators, other nanophotonic platforms have the potential to further enhance the nonlinear effects. For example, high-density atom gases confined in waveguides may significantly increase photon interactions [109-112]. In Ref. [110], a third-order susceptibility as large as $\sim 10^{-12} \mathrm{~m}^{2} / \mathrm{V}^{2}$ is calculated, which gives $U \sim$ $1 \mathrm{GHz}$ when the effective mode volume is $\sim 10^{3} \mu \mathrm{m}^{3}$. Additionally, arbitrarily long-range coupling in synthetic frequency dimensions is also possible by special designs of the electrooptic modulation profile [113].

In conclusion, we systematically develop a theoretical framework on the creation of the $N$-boson synthetic lattice that enlightens insights into the studies of both synthetic dimensions and the physics of multiple interactive indistinguishable bosons. The connection between multiple bosons and multiple dimensions is highlighted. Through band structure analysis, interesting dynamics of multiboson states is unveiled, and can be confirmed by numerical simulations. Our study hence points out an alternative way towards the studies of bosonboson interactions and multiboson dynamics on lattices, and also holds potentials for exploring important multiboson manipulations together with nonlinearity, nontrivial topology, and/or boson entanglements with possible applications in the fields of quantum computations, quantum simulations, and quantum information processing.

\section{ACKNOWLEDGMENTS}

The research is supported by National Natural Science Foundation of China (11974245), National Key R\&D Program of China (2017YFA0303701), Shanghai Municipal Science and Technology Major Project (2019SHZDZX01), and Natural Science Foundation of Shanghai (19ZR1475700). L.Y. acknowledges support from the Program for Professor of Special Appointment (Eastern Scholar) at Shanghai Institutions of Higher Learning. X.C. also acknowledges the support from Shandong Quancheng Scholarship (Award No. 00242019024). This work was also partially supported by the Fundamental Research Funds for the Central Universities.

\section{APPENDIX A: EXCHANGE SYMMETRY AND LATTICE UNIFORMITY}

In the main text, we show that Eq. (9) is converted by the exchange symmetry to Eq. (13) which features translational symmetry. Here, we consider another symmetry restriction of the wave function that $v_{\lambda_{1}, \ldots, \lambda_{N}}=0$ except in the region $\lambda_{1} \leqslant \lambda_{2} \leqslant \cdots \leqslant \lambda_{N}$, so that the number of $v_{\lambda_{1}, \ldots, \lambda_{N}}$ variables with nonzero values is minimum. We claim that with this restriction, Eq. (13) does not hold. Actually, by assigning $\lambda_{i}=$ $\lambda_{0}(1 \leqslant i \leqslant N-1)$ and $\lambda_{N}=\lambda_{0}+1$, we obtain from Eq. (9) that

$$
\begin{aligned}
& i \dot{v}_{\lambda_{0}, \lambda_{0}, \ldots, \lambda_{0}, \lambda_{0}+1} \\
& =g(N-1) !\left(2 v_{\lambda_{0}, \lambda_{0}, \ldots, \lambda_{0}+1, \lambda_{0}+1}+v_{\lambda_{0}, \lambda_{0}, \ldots, \lambda_{0}, \lambda_{0}+2}\right. \\
& \left.\quad+N v_{\lambda_{0}, \lambda_{0}, \ldots, \lambda_{0}, \lambda_{0}}+v_{\lambda_{0}-1, \lambda_{0}, \ldots, \lambda_{0}, \lambda_{0}+1}\right) .
\end{aligned}
$$

On the other hand, if Eq. (13) were still valid, it should take the form

$$
\begin{aligned}
i \dot{v}_{\lambda_{0}, \lambda_{0}, \ldots, \lambda_{0}, \lambda_{0}+1}= & g\left(v_{\lambda_{0}, \lambda_{0}, \ldots, \lambda_{0}+1, \lambda_{0}+1}+v_{\lambda_{0}, \lambda_{0}, \ldots, \lambda_{0}, \lambda_{0}+2}\right. \\
& \left.+v_{\lambda_{0}, \lambda_{0}, \ldots, \lambda_{0}, \lambda_{0}}+v_{\lambda_{0}-1, \lambda_{0}, \ldots, \lambda_{0}, \lambda_{0}+1}\right) .
\end{aligned}
$$

Immediately one notices that Eqs. (A1) and (A2) are contradictory. 
(a)

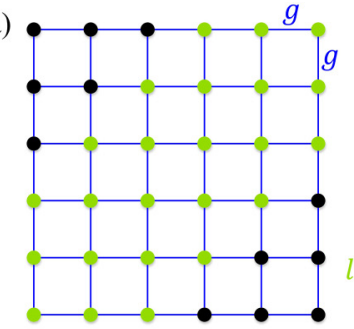

(d)

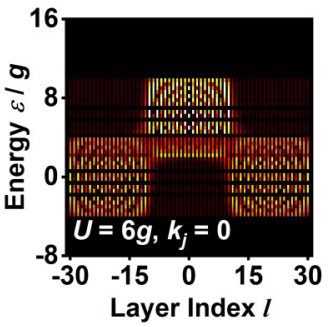

(e)

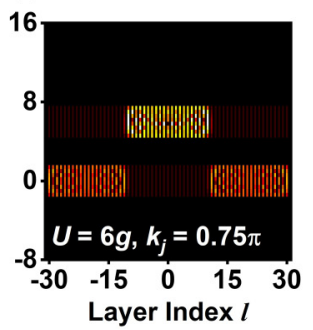

(b)

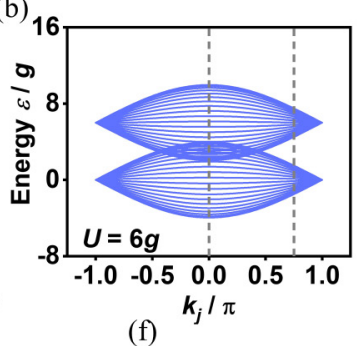

(f)

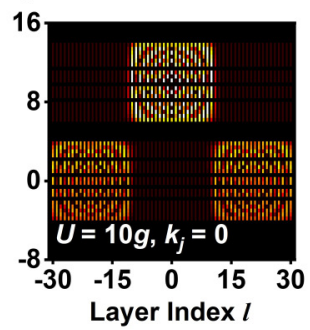

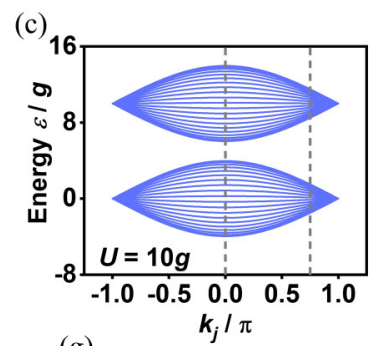

(g)

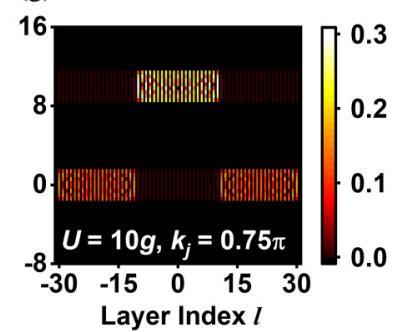

FIG. 15. The two-boson tight-binding synthetic lattice with interactions. (a) The two-boson synthetic lattice with the exchange symmetry restriction on the wave function $v_{m n}(t)$. Green spots indicate nonzero on-site potentials. (b), (c) Projected band structures of the lattice in (a) with $U=6 g$ or $10 g$. (d) $-(\mathrm{g})$ Eigenstate distributions corresponding to the band structures in (b), (c), with $U=6 g$ or $10 g$ and $k_{j}=0$ or $0.75 \pi$ [gray dashes in (b), (c)]. $R=10$ and $-30 \leqslant l \leqslant 30$ in (b) $-(\mathrm{g})$.

For the two-boson case $(N=2)$, if we require that $v_{m n}=0$ when $n<m$, Eq. (3) in the main text is simplified to

$$
\begin{aligned}
i \dot{v}_{m m}= & g\left(v_{m-1, m}+v_{m, m+1}\right), \\
i \dot{v}_{m, m+1}= & g\left(v_{m-1, m+1}+2 v_{m+1, m+1}+2 v_{m m}+v_{m, m+2}\right), \\
i \dot{v}_{m n}= & g\left(v_{m-1, n}+v_{m+1, n}+v_{m, n-1}+v_{m, n+1}\right) \\
& \text { if } n \geqslant m+2,
\end{aligned}
$$

while with this restriction Eq. (7) states that

$$
\begin{aligned}
i \dot{v}_{m m}= & g\left(v_{m-1, m}+v_{m, m+1}\right), \\
i \dot{v}_{m, m+1}= & g\left(v_{m-1, m+1}+v_{m+1, m+1}+v_{m m}+v_{m, m+2}\right), \\
i \dot{v}_{m n}= & g\left(v_{m-1, n}+v_{m+1, n}+v_{m, n-1}+v_{m, n+1}\right) \\
& \text { if } n \geqslant m+2 .
\end{aligned}
$$

The discrepancies in Eqs. (A3) and (A4) are irreconcilable, which indicates that Eq. (7) is incorrect when we choose $v_{m n}=0(n<m)$. Without the translational symmetry in Eq. (7), the Fourier transform cannot be performed mathematically, and thus the band structure analysis in the two-boson synthetic lattice fails. As an additional illustration, Fig. 13(a) shows the lattice structure related to Eq. (A3), where the coupling strengths are not uniform throughout the lattice.

\section{APPENDIX B: TWO BOSONS ON A TIGHT-BINDING LATTICE WITH INTERACTIONS}

Consider the following extended Bose-Hubbard Hamiltonian involving a nonlocal, nonlinear interactive term:

$$
H=g \sum_{k=-\infty}^{+\infty}\left(b_{k}^{\dagger} b_{k+1}+b_{k+1}^{\dagger} b_{k}\right)+\frac{U}{2} \sum_{k=-\infty}^{+\infty} \sum_{s=-R}^{R} b_{k+s}^{\dagger} b_{k}^{\dagger} b_{k} b_{k+s} .
$$

The two-boson state is the same as Eq. (2), and the dynamics of the $v_{m n}$ 's derived from the Schrödinger equation $i|\dot{\phi}\rangle=H|\phi\rangle$ reads (assuming $R \geqslant 2$ )

$$
\begin{aligned}
i\left(\dot{v}_{m n}+\dot{v}_{n m}\right)= & g\left(v_{m-1, n}+v_{m+1, n}+v_{m, n-1}+v_{m, n+1}\right. \\
& \left.+v_{n-1, m}+v_{n+1, m}+v_{n, m-1}+v_{n, m+1}\right) \\
& +U \delta_{|m-n| \leqslant R}\left(v_{m n}+v_{n m}\right),
\end{aligned}
$$

where $\delta_{|m-n| \leqslant R}$ is defined as in Eq. (28). If the restriction that $v_{m n}=0$ when $n<m$ is used, Eq. (B2) is mapped to the nonuniform lattice structure in Fig. 13(b); if the exchange symmetry $\left(v_{m n}=v_{n m}\right)$ is applied, nevertheless, Eq. (B2) is converted to

$$
\begin{aligned}
i \dot{v}_{m n}= & g\left(v_{m-1, n}+v_{m, n-1}+v_{m+1, n}+v_{m, n+1}\right) \\
& +U \delta_{|m-n| \leqslant R} v_{m n} .
\end{aligned}
$$

Equation (B3) corresponds to the two-boson synthetic lattice in Fig. 2(a), which possesses translational symmetry along the $j$ axis ( $m=n$ direction) and the wave vector $k_{j}=$ $k_{m}+k_{n}$. The projected band structure $\varepsilon\left(k_{j}\right)$ can be plotted for different parameters $g$ and $U$. The case of $U=0$ is shown in Fig. 8(b), which can be viewed as the 2D tight-binding band structure [Fig. 8(a)] after projection along the $\Gamma-M$ direction in the first Brillouin zone and then band folding. Figures 15(b) and $15(\mathrm{c})$, respectively, show the projected band structure when $U=6 \mathrm{~g}$ and $U=10 \mathrm{~g}$. It is obvious that compared to the case of $U=0$, when $U>0$, some of the bands located around energy $\varepsilon=0$ are lifted to higher-energy positions, and when $U>8 g$, the lifted and unlifted bands do not overlap, thus forming a "band gap" in between. The eigenstate distributions are further explored at different values of $U$ and $k_{j}$, and the results are displayed in Figs. 15(d)-15(g). It is shown that the lifted bands at higher energy correspond to eigenstates located in the nonlinear region around $l=0$ (or equivalently 
(a)

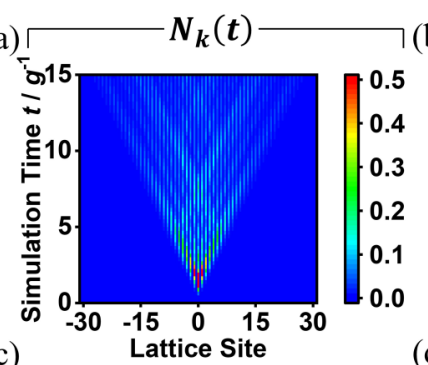

(c)

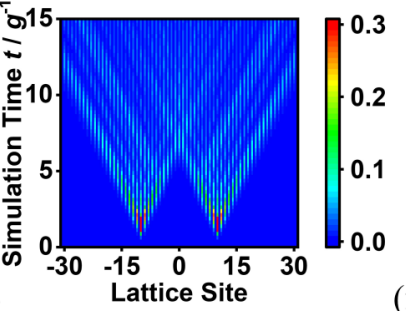

(e)

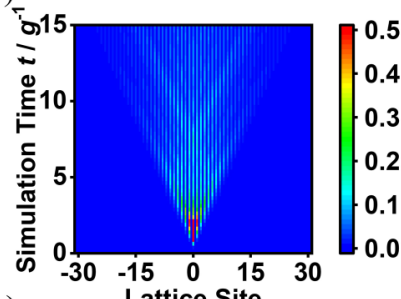

(g)

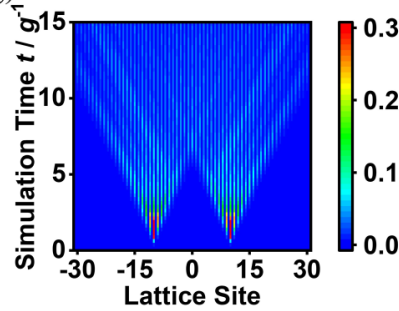

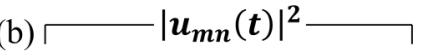

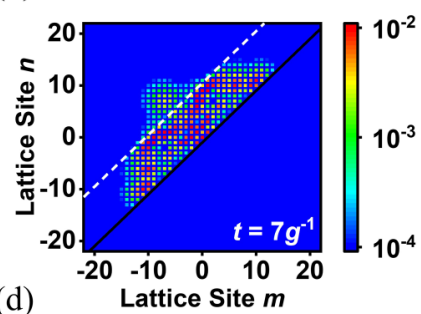

(d)
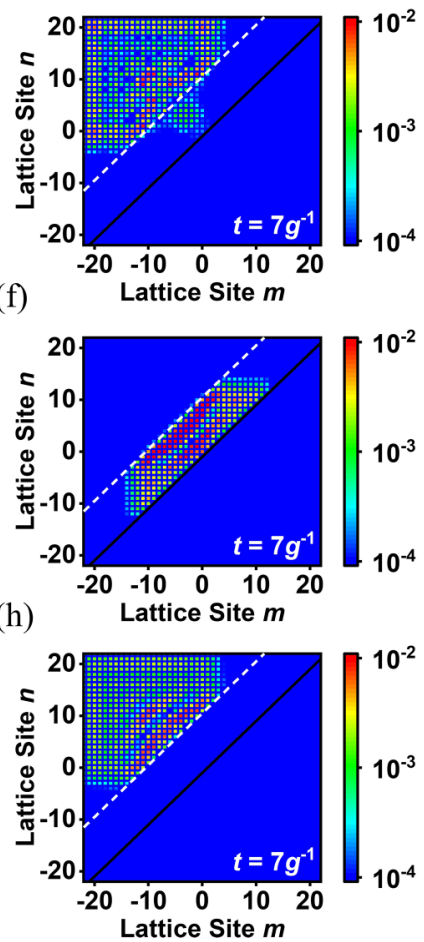

FIG. 16. Simulation results of the collapse/isolation of twoboson bound states and scattering states in the two-boson tightbinding synthetic lattice with interactions. (a), (b) The wave packet evolution after exciting two bosons on the 0th site simultaneously with $U=6 g$ and $\Delta \varepsilon=3 g$. (c), (d) The wave packet evolution after exciting two bosons on the -10 th and 10th sites, respectively, with $U=6 g$ and $\Delta \varepsilon=3 g$. (e), (f) The wave packet evolution after exciting two bosons on the 0th site simultaneously with $U=10 \mathrm{~g}$ and $\Delta \varepsilon=5 g$. (g), (h) The wave packet evolution after exciting two bosons on the -10 th and 10th sites, respectively, with $U=10 \mathrm{~g}$ and $\Delta \varepsilon=5 g . R=10, t_{0}=g^{-1}$, and $\tau^{2}=0.375 g^{-2}$ in this figure.

the $m=n$ direction, usually referred to as two-boson bound states), and the unlifted bands correspond to the linear region (referred to as scattering states). This further confirms that the nonlinear interactive term in Eq. (B1) renders an on-site potential added to a stripe $|m-n| \leqslant R$ in the two-boson synthetic lattice [Fig. 15(a)].

The appearance of a band gap is a reminder here that the dynamics of two-boson states might be influenced by the value of $U / g$. To be more specific, it might be predicted that when $U=6 g$, the bound (nonlinear) and scattering (linear) states are "connected" by the eigenstates whose energies are within the overlapping range $U-4 g<\varepsilon<4 g$, so that the bound states and scattering states may collapse into each other. (Interestingly, only the collapse from bound states into scattering states was mentioned in previous studies [37], while the collapse process in the opposite direction was not paid attention to.) When $U=10 g$, the linear and nonlinear regions in the lattice are isolated because of the existence of a band gap. Any state with an energy inside this band gap is forbidden. This prediction is confirmed by our numerical results of $N_{k}(t)$, the average boson number on the $k$ th site of the 1D tight-binding chain, as well as $\left|u_{m n}(t)\right|^{2}$, the probability to find one boson on the $m$ th site and the other on the $n$th site (Fig. 16). In Fig. 16, Eq. (B3) is solved under input-output formalism with $R=10$. When $U=6 g$ and lattice site $(0,0)$ is excited with energy $\Delta \varepsilon=3 g$, as shown in Fig. 16(b), the two-boson bound state "leaks" into the linear region above the white dashed line. When $U=6 \mathrm{~g}$ and the excitation source is placed at $(-10,10)$, which is in the linear lattice region, significant excitation probabilities on nonlinear sites are also clearly observed [Fig. 16(d)]. When $U=10 g$, however, the excited two-boson state is strictly localized in the region where it is initially excited, since the lifted and unlifted bands do not overlap. In fact, $U=8 g$ is the collapse/isolation threshold of two-boson bound states and scattering states. Finally, it should be pointed out that the differences of simulation results between $U=6 g$ and $U=10 g$ are not significant if only the average boson numbers on lattice sites $\left[N_{k}(t)\right]$ are resolved [Fig. 16(a) vs 16(e), 16(c) vs $16(\mathrm{~g})$ ], suggesting that twoboson correlated detection [direct measurement of $\left|u_{m n}(t)\right|^{2}$ ] might be necessary to reveal the distinctions between $U=6 g$ and $U=10 g$.
[1] A. P. Schnyder, S. Ryu, A. Furusaki, and A. W. W. Ludwig, Classification of topological insulators and superconductors in three spatial dimensions, Phys. Rev. B 78, 195125 (2008).

[2] A. Kitaev, Periodic table for topological insulators and superconductors, in Advances in Theoretical Physics: Landau Memorial Conference, AIP Conf. Proc. No. 1134 (AIP, Melville, NY, 2009), p. 22.

[3] S. Ryu, A. P. Schnyder, A. Furusaki, and A. W. W. Ludwig, Topological insulators and superconductors: Tenfold way and dimensional hierarchy, New J. Phys. 12, 065010 (2010).

[4] M. Z. Hasan and C. L. Kane, Colloquium: Topological insulators, Rev. Mod. Phys. 82, 3045 (2010).
[5] E. Lustig and M. Segev, Topological photonics in synthetic dimensions, Adv. Opt. Photonics 13, 426 (2021).

[6] Z. Chen and M. Segev, Highlighting photonics: Looking into the next decade, eLight 1, 2 (2021).

[7] S. T. Ruggiero, T. W. Barbee, Jr., and M. R. Beasley, Superconductivity in Quasi-Two-Dimensional Layered Composites, Phys. Rev. Lett. 45, 1299 (1980).

[8] Y. Guo, Y.-F. Zhang, X.-Y. Bao, T.-Z. Han, Z. Tang, L.-X. Zhang, W.-G. Zhu, E. G. Wang, Q. Niu, Z. Q. Qiu et al., Superconductivity modulated by quantum size effects, Science 306, 1915 (2004). 
[9] K. Y. Arutyunov, D. S. Golubev, and A. D. Zaikin, Superconductivity in one dimension, Phys. Rep. 464, 1 (2008).

[10] S. Qin, J. Kim, Q. Niu, and C.-K. Shih, Superconductivity at the two-dimensional limit, Science 324, 1314 (2009).

[11] J. Guo, Y. Zhou, C. Huang, S. Cai, Y. Sheng, G. Gu, C. Yang, G. Lin, K. Yang, A. Li et al., Crossover from two-dimensional to three-dimensional superconducting states in bismuth-based cuprate superconductor, Nat. Phys. 16, 295 (2020).

[12] A. Celi, P. Massignan, J. Ruseckas, N. Goldman, I. B. Spielman, G. Juzeliūnas, and M. Lewenstein, Synthetic Gauge Fields in Synthetic Dimensions, Phys. Rev. Lett. 112, 043001 (2014).

[13] M. Mancini, G. Pagano, G. Cappellini, L. Livi, M. Rider, J. Catani, C. Sias, P. Zoller, M. Inguscio, M. Dalmonte et al., Observation of chiral edge states with neutral fermions in synthetic Hall ribbons, Science 349, 1510 (2015).

[14] T. Ozawa, H. M. Price, N. Goldman, O. Zilberberg, and I. Carusotto, Synthetic dimensions in integrated photonics: From optical isolation to four-dimensional quantum Hall physics, Phys. Rev. A 93, 043827 (2016).

[15] L. Yuan, Q. Lin, M. Xiao, and S. Fan, Synthetic dimension in photonics, Optica 5, 1396 (2018).

[16] E. Lustig, S. Weimann, Y. Plotnik, Y. Lumer, M. A. Bandres, A. Szameit, and M. Segev, Photonic topological insulator in synthetic dimensions, Nature (London, UK) 567, 356 (2019).

[17] A. Dutt, Q. Lin, L. Yuan, M. Minkov, M. Xiao, and S. Fan, A single photonic cavity with two independent physical synthetic dimensions, Science 367, 59 (2020).

[18] Z. Liu, Q. Zhang, F. Qin, D. Zhang, X. Liu, and J. J. Xiao, Surface states ensured by a synthetic Weyl point in one-dimensional plasmonic dielectric crystals with broken inversion symmetry, Phys. Rev. B 99, 085441 (2019).

[19] Z. Liu, Q. Zhang, F. Qin, D. Zhang, X. Liu, and J. J. Xiao, Type-II Dirac point and extreme dispersion in one-dimensional plasmonic-dielectric crystals with off-axis propagation, Phys. Rev. A 99, 043828 (2019).

[20] T.-S. Zeng, C. Wang, and H. Zhai, Charge Pumping of Interacting Fermion Atoms in the Synthetic Dimension, Phys. Rev. Lett. 115, 095302 (2015).

[21] L. F. Livi, G. Cappellini, M. Diem, L. Franchi, C. Clivati, M. Frittelli, F. Levi, D. Calonico, J. Catani, M. Inguscio et al., Synthetic Dimensions and Spin-Orbit Coupling with an Optical Clock Transition, Phys. Rev. Lett. 117, 220401 (2016).

[22] H. M. Price, T. Ozawa, and H. Schomerus, Synthetic dimensions and topological chiral currents in mesoscopic rings, Phys. Rev. Research 2, 032017(R) (2020).

[23] J. Wang, J.-Y. Yang, I. M. Fazal, N. Ahmed, Y. Yan, H. Huang, Y. Ren, Y. Yue, S. Dolinar, M. Tur et al., Terabit free-space data transmission employing orbital angular momentum multiplexing, Nat. Photonics 6, 488 (2012)

[24] M. Mirhosseini, O. S. Magaña-Loaiza, M. N. O’Sullivan, B. Rodenburg, M. Malik, M. P. J. Lavery, M. J. Padgett, D. J. Gauthier, and R. W. Boyd, High-dimensional quantum cryptography with twisted light, New J. Phys. 17, 033033 (2015).

[25] H.-H. Lu, J. M. Lukens, N. A. Peters, O. D. Odele, D. E. Leaird, A. M. Weiner, and P. Lougovski, Electro-Optic Frequency Beam Splitters and Tritters for High-Fidelity Photonic Quantum Information Processing, Phys. Rev. Lett. 120, 030502 (2018).
[26] C. Joshi, A. Farsi, S. Clemmen, S. Ramelow, and A. L. Gaeta, Frequency multiplexing for quasi-deterministic heralded single-photon sources, Nat. Commun. 9, 847 (2018).

[27] S. Buddhiraju, A. Dutt, M. Minkov, I. A. D. Williamson, and S. Fan, Arbitrary linear transformations for photons in the frequency synthetic dimension, Nat. Commun. 12, 2401 (2021).

[28] A. Aspuru-Guzik and P. Walther, Photonic quantum simulators, Nat. Phys. 8, 285 (2012).

[29] A. Schreiber, A. Gábris, P. P. Rohde, K. Laiho, M. Štefaňák, V. Potoček, C. Hamilton, I. Jex, and C. Silberhorn, A 2D quantum walk simulation of two-particle dynamics, Science 336, 55 (2012).

[30] T. J. Sturges, T. McDermott, A. Buraczewski, W. R. Clements, J. J. Renema, S. W. Nam, T. Gerrits, A. Lita, W. S. Kolthammer, A. Eckstein et al., Quantum simulations with multiphoton Fock states, npj Quantum Inf. 7, 91 (2021).

[31] K. Winkler, G. Thalhammer, F. Lang, R. Grimm, J. Hecker Denschlag, A. J. Daley, A. Kantian, H. P. Büchler, and P. Zoller, Repulsively bound atom pairs in an optical lattice, Nature (London, UK) 441, 853 (2006).

[32] M. Valiente and D. Petrosyan, Two-particle states in the Hubbard model, J. Phys. B: At., Mol. Opt. Phys. 41, 161002 (2008).

[33] M. Valiente and D. Petrosyan, Quantum dynamics of one and two bosonic atoms in a combined tight-binding periodic and weak parabolic potential, Europhys. Lett. 83, 30007 (2008).

[34] S. Longhi and G. Della Valle, Tamm-Hubbard surface states in the continuum, J. Phys. Condens. Matter 25, 235601 (2013).

[35] M. Valiente and D. Petrosyan, Scattering resonances and twoparticle bound states of the extended Hubbard model, J. Phys. B: At., Mol. Opt. Phys. 42, 121001 (2009).

[36] M. Valiente, Lattice two-body problem with arbitrary finiterange interactions, Phys. Rev. A 81, 042102 (2010).

[37] M. A. Gorlach and A. N. Poddubny, Interaction-induced twophoton edge states in an extended Hubbard model realized in a cavity array, Phys. Rev. A 95, 033831 (2017).

[38] M. Di Liberto, A. Recati, I. Carusotto, and C. Menotti, Twobody physics in the Su-Schrieffer-Heeger model, Phys. Rev. A 94, 062704 (2016).

[39] M. A. Gorlach and A. N. Poddubny, Topological edge states of bound photon pairs, Phys. Rev. A 95, 053866 (2017).

[40] M. Di Liberto, A. Recati, I. Carusotto, and C. Menotti, Two-body bound and edge states in the extended SSH BoseHubbard model, Eur. Phys. J.: Spec. Top. 226, 2751 (2017).

[41] A. M. Marques and R. G. Dias, Topological bound states in interacting Su-Schrieffer-Heeger rings, J. Phys. Condens. Matter 30, 305601 (2018).

[42] A. A. Stepanenko and M. A. Gorlach, Interaction-induced topological states of photon pairs, Phys. Rev. A 102, 013510 (2020).

[43] R. Khomeriki, D. O. Krimer, M. Haque, and S. Flach, Interaction-induced fractional Bloch and tunneling oscillations, Phys. Rev. A 81, 065601 (2010).

[44] S. Longhi, Photonic Bloch oscillations of correlated particles, Opt. Lett. 36, 3248 (2011).

[45] G. Corrielli, A. Crespi, G. Della Valle, S. Longhi, and R. Osellame, Fractional Bloch oscillations in photonic lattices, Nat. Commun. 4, 1555 (2013). 
[46] S.-y. Chu and D.-c. Su, Operation of a two-mode laser in a three-level atomic system with a common upper level, Phys. Rev. A 25, 3169 (1982).

[47] D.-W. Wang, H. Cai, R.-B. Liu, and M. O. Scully, Mesoscopic Superposition States Generated by Synthetic Spin-Orbit Interaction in Fock-State Lattices, Phys. Rev. Lett. 116, 220502 (2016).

[48] K. Tschernig, R. de J. León-Montiel, A. Pérez-Leija, and K. Busch, Multiphoton synthetic lattices in multiport waveguide arrays: Synthetic atoms and Fock graphs, Photonics Res. 8, 1161 (2020).

[49] H. Cai and D.-W. Wang, Topological phases of quantized light, Natl. Sci. Rev. 8, nwaa196 (2021).

[50] A. Yariv, Coupled-mode theory for guided-wave optics, IEEE J. Quantum Electron. 9, 919 (1973).

[51] A. P. Sutton, M. W. Finnis, D. G. Pettifor, and Y. Ohta, The tight-binding bond model, J. Phys. C: Solid State Phys. 21, 35 (1988).

[52] S. Fan, Ş. E. Kocabaş, and J.-T. Shen, Input-output formalism for few-photon transport in one-dimensional nanophotonic waveguides coupled to a qubit, Phys. Rev. A 82, 063821 (2010).

[53] W. P. Su, J. R. Schrieffer, and A. J. Heeger, Solitons in Polyacetylene, Phys. Rev. Lett. 42, 1698 (1979).

[54] E. J. Meier, F. A. An, and B. Gadway, Observation of the topological soliton state in the Su-Schrieffer-Heeger model, Nat. Commun. 7, 13986 (2016).

[55] S. Liu, W. Gao, Q. Zhang, S. Ma, L. Zhang, C. Liu, Y. J. Xiang, T. J. Cui, and S. Zhang, Topologically protected edge state in two-dimensional Su-Schrieffer-Heeger circuit, Research 2019, 8609875 (2019).

[56] L.-Y. Zheng, V. Achilleos, O. Richoux, G. Theocharis, and V. Pagneux, Observation of Edge Waves in a Two-Dimensional Su-Schrieffer-Heeger Acoustic Network, Phys. Rev. Appl. 12, 034014 (2019).

[57] W. Zhang and X. Zhang, Quadrupole topological phases in the zero-dimensional optical cavity, Europhys. Lett. 131, 24004 (2020).

[58] A. Dutt, M. Minkov, I. A. D. Williamson, and S. Fan, Higherorder topological insulators in synthetic dimensions, Light Sci. Appl. 9, 131 (2020).

[59] M. Kim and J. Rho, Topological edge and corner states in a two-dimensional photonic Su-Schrieffer-Heeger lattice, Nanophotonics 9, 3227 (2020).

[60] F. Liu and K. Wakabayashi, Novel Topological Phase with a Zero Berry Curvature, Phys. Rev. Lett. 118, 076803 (2017).

[61] D. Obana, F. Liu, and K. Wakabayashi, Topological edge states in the Su-Schrieffer-Heeger model, Phys. Rev. B 100, 075437 (2019).

[62] A. Coutant, V. Achilleos, O. Richoux, G. Theocharis, and V. Pagneux, Robustness of topological corner modes against disorder with application to acoustic networks, Phys. Rev. B 102, 214204 (2020).

[63] M. Valiente, D. Petrosyan, and A. Saenz, Three-body bound states in a lattice, Phys. Rev. A 81, 011601(R) (2010).

[64] A. Miranowicz, M. Paprzycka, Y.-x. Liu, J. Bajer, and F. Nori, Two-photon and three-photon blockades in driven nonlinear systems, Phys. Rev. A 87, 023809 (2013).

[65] Q.-Y. Liang, A. V. Venkatramani, S. H. Cantu, T. L. Nicholson, M. J. Gullans, A. V. Gorshkov, J. D. Thompson, C. Chin,
M. D. Lukin, and V. Vuletić, Observation of three-photon bound states in a quantum nonlinear medium, Science 359, 783 (2018).

[66] A. Yariv, Y. Xu, R. K. Lee, and A. Scherer, Coupled-resonator optical waveguide: A proposal and analysis, Opt. Lett. 24, 711 (1999).

[67] M. J. Hartmann, F. G. S. L. Brandão, and M. B. Plenio, Strongly interacting polaritons in coupled arrays of cavities, Nat. Phys. 2, 849 (2006).

[68] M. J. Hartmann, F. G. S. L. Brandão, and M. B. Plenio, Quantum many-body phenomena in coupled cavity arrays, Laser Photonics Rev. 2, 527 (2008).

[69] R. O. Umucalılar and I. Carusotto, Artificial gauge field for photons in coupled cavity arrays, Phys. Rev. A 84, 043804 (2011).

[70] D. O. Krimer and R. Khomeriki, Realization of discrete quantum billiards in a two-dimensional optical lattice, Phys. Rev. A 84, 041807(R) (2011).

[71] L. Yuan, Y. Shi, and S. Fan, Photonic gauge potential in a system with a synthetic frequency dimension, Opt. Lett. 41, 741 (2016).

[72] W.-g. Song, H. Li, S. Gao, C. Chen, S. Zhu, and T. Li, Subwavelength self-imaging in cascaded waveguide arrays, Adv. Photonics 2, 036001 (2020).

[73] J. Zhang, V. Pelgrin, C. Alonso-Ramos, L. Vivien, S. He, and E. Cassan, Stretching the spectra of Kerr frequency combs with self-adaptive boundary silicon waveguides, Adv. Photonocs 2, 046001 (2020).

[74] F. Klauck, M. Heinrich, and A. Szameit, Photonic two-particle quantum walks in Su-Schrieffer-Heeger lattices, Photonics Res. 9, A1 (2021).

[75] D. Jaksch, C. Bruder, J. I. Cirac, C. W. Gardiner, and P. Zoller, Cold Bosonic Atoms in Optical Lattices, Phys. Rev. Lett. 81, 3108 (1998).

[76] R. Grimm, M. Weidemüller, and Y. B. Ovchinnikov, Optical dipole traps for neutral atoms, Adv. At., Mol. Opt. Phys. 42, 95 (2000).

[77] I. Bloch, J. Dalibard, and W. Zwerger, Many-body physics with ultracold gases, Rev. Mod. Phys. 80, 885 (2008).

[78] C. Chin, R. Grimm, P. Julienne, and E. Tiesinga, Feshbach resonances in ultracold gases, Rev. Mod. Phys. 82, 1225 (2010).

[79] L. Zeng and J. Zeng, Gap-type dark localized modes in a Bose-Einstein condensate with optical lattices, Adv. Photonics 1, 046004 (2019).

[80] M. H. Devoret and R. J. Schoelkopf, Superconducting circuits for quantum information: an outlook, Science 339, 1169 (2013).

[81] X. Gu, S. Chen, and Y.-x. Liu, Topological edge states and pumping in a chain of coupled superconducting qubits, arXiv:1711.06829.

[82] Z. Yan, Y.-R. Zhang, M. Gong, Y. Wu, Y. Zheng, S. Li, C. Wang, F. Liang, J. Lin, Y. Xu et al., Strongly correlated quantum walks with a 12-qubit superconducting processor, Science 364, 753 (2019).

[83] Y. Yanay, J. Braumüller, S. Gustavsson, W. D. Oliver, and C. Tahan, Two-dimensional hard-core Bose-Hubbard model with superconducting qubits, npj Quantum Inf. 6, 58 (2020).

[84] A. Akhieser, L. Landau, and I. Pomeranchook, Scattering of light by light, Nature (London, UK) 138, 206 (1936). 
[85] O. Morsch and M. Oberthaler, Dynamics of Bose-Einstein condensates in optical lattices, Rev. Mod. Phys. 78, 179 (2006).

[86] D. C. McKay and B. DeMarco, Cooling in strongly correlated optical lattices: Prospects and challenges, Rep. Prog. Phys. 74, 054401 (2011).

[87] C. Xu, Z.-G. Chen, G. Zhang, G. Ma, and Y. Wu, Multi-dimensional wave steering with higher-order topological phononic crystal, Sci. Bull. (2021), doi: 10.1016/j.scib.2021.05.013.

[88] L. Yuan and S. Fan, Bloch oscillation and unidirectional translation of frequency in a dynamically modulated ring resonator, Optica 3, 1014 (2016).

[89] A. Dutt, M. Minkov, Q. Lin, L. Yuan, D. A. B. Miller, and S. Fan, Experimental band structure spectroscopy along a synthetic dimension, Nat. Commun. 10, 3122 (2019).

[90] G. Li, Y. Zheng, A. Dutt, D. Yu, Q. Shan, S. Liu, L. Yuan, S. Fan, and X. Chen, Dynamic band structure measurement in the synthetic space, Sci. Adv. 7, eabe4335 (2021).

[91] L. D. Tzuang, M. Soltani, Y. H. D. Lee, and M. Lipson, High RF carrier frequency modulation in silicon resonators by coupling adjacent free-spectral-range modes, Opt. Lett. 39, 1799 (2014).

[92] P. O. Weigel, J. Zhao, K. Fang, H. Al-Rubaye, D. Trotter, D. Hood, J. Mudrick, C. Dallo, A. T. Pomerene, A. L. Starbuck et al., Bonded thin film lithium niobate modulator on a silicon photonics platform exceeding $100 \mathrm{GHz} 3-\mathrm{dB}$ electrical modulation bandwidth, Opt. Express 26, 23728 (2018).

[93] L. Yuan, Q. Lin, A. Zhang, M. Xiao, X. Chen, and S. Fan, Photonic Gauge Potential in One Cavity with Synthetic Frequency and Orbital Angular Momentum Dimensions, Phys. Rev. Lett. 122, 083903 (2019).

[94] E. Dulkeith, F. Xia, L. Schares, W. M. J. Green, and Y. A. Vlasov, Group index and group velocity dispersion in siliconon-insulator photonic wires, Opt. Express 14, 3853 (2006).

[95] A. C. Turner, C. Manolatou, B. S. Schmidt, M. Lipson, M. A. Foster, J. E. Sharping, and A. L. Gaeta, Tailored anomalous group-velocity dispersion in silicon channel waveguides, Opt. Express 14, 4357 (2006).

[96] J. T. Shen and S. Fan, Coherent photon transport from spontaneous emission in one-dimensional waveguides, Opt. Lett. 30, 2001 (2005).

[97] J. D. Thompson, T. G. Tiecke, N. P. de Leon, J. Feist, A. V. Akimov, M. Gullans, A. S. Zibrov, V. Vuletić, and M. D. Lukin, Coupling a single trapped atom to a nanoscale optical cavity, Science 340, 1202 (2013).

[98] A. Goban, C. L. Hung, S. P. Yu, J. D. Hood, J. A. Muniz, J. H. Lee, M. J. Martin, A. C. McClung, K. S. Choi, D. E. Chang et al., Atom-light interactions in photonic crystals, Nat. Commun. 5, 3808 (2014).
[99] R. Zektzer, E. Talker, Y. Barash, N. Mazurski, L. Stern, and U. Levy, Atom-photon interactions in atomic cladded waveguides: Bridging atomic and telecom technologies, ACS Photonics 8, 879 (2021).

[100] R. Bruck and O. L. Muskens, Plasmonic nanoantennas as integrated coherent perfect absorbers on SOI waveguides for modulators and all-optical switches, Opt. Express 21, 27652 (2013).

[101] H. Park, S.-Y. Lee, J. Kim, B. Lee, and H. Kim, Near-infrared coherent perfect absorption in plasmonic metal-insulatormetal waveguide, Opt. Express 23, 24464 (2015).

[102] R. Soref, The past, present, and future of silicon photonics, IEEE J. Sel. Top. Quantum Electron. 12, 1678 (2006).

[103] C. T. Phare, Y.-H. Daniel Lee, J. Cardenas, and M. Lipson, Graphene electro-optic modulator with $30 \mathrm{GHz}$ bandwidth, Nat. Photonics 9, 511 (2015).

[104] J.-C. Weeber, G. Colas-des-Francs, A. Bouhelier, A. Leray, K. Vasilev, X. Yu, K. Hammani, J.-M. Arocas, G. Gadret, L. Markey et al., Colloidal quantum dots decorated microring resonators for efficient integrated waveguides excitation, Nanophotonics 9, 1411 (2020).

[105] B. E. Little, S. T. Chu, H. A. Haus, J. Foresi, and J. Laine, Microring resonator channel dropping filters, J. Light. Technol. 15, 998 (1997).

[106] B. E. Little, S. T. Chu, P. P. Absil, J. V. Hryniewicz, F. G. Johnson, F. Seiferth, D. Gill, V. Van, O. King, and M. Trakalo, Very high-order microring resonator filters for WDM applications, IEEE Photonics Technol. Lett. 16, 2263 (2004).

[107] D. G. Rabus, Integrated Ring Resonators (Springer, Berlin, 2007).

[108] L. Yuan, A. Dutt, M. Qin, S. Fan, and X. Chen, Creating locally interacting Hamiltonians in the synthetic frequency dimension for photons, Photonics Res. 8, B8 (2020).

[109] C. Perrella, P. S. Light, J. D. Anstie, F. Benabid, T. M. Stace, A. G. White, and A. N. Luiten, High-efficiency cross-phase modulation in a gas-filled waveguide, Phys. Rev. A 88, 013819 (2013).

[110] V. Venkataraman, K. Saha, and A. L. Gaeta, Phase modulation at the few-photon level for weak-nonlinearity-based quantum computing, Nat. Photonics 7, 138 (2013).

[111] L. Stern, B. Desiatov, N. Mazurski, and U. Levy, Strong coupling and high-contrast all-optical modulation in atomic cladding waveguides, Nat. Commun. 8, 14461 (2017).

[112] D. Roy, C. M. Wilson, and O. Firstenberg, Colloquium: Strongly interacting photons in one-dimensional continuum, Rev. Mod. Phys. 89, 021001 (2017).

[113] L. Yuan, M. Xiao, Q. Lin, and S. Fan, Synthetic space with arbitrary dimensions in a few rings undergoing dynamic modulation, Phys. Rev. B 97, 104105 (2018). 